\title{
Analysis of phenolic compounds in leaves from endemic trees from Madeira Island. A contribution to the chemotaxonomy of Laurisilva forest species
}

\author{
Eulogio J. Llorent-Martínez ${ }^{\mathrm{a}, \mathrm{b}}$, Sandra Gouveia ${ }^{\mathrm{a}}$, Paula C. Castilho ${ }^{\mathrm{a}, *}$ \\ a Centro de Química da Madeira (CQM), Centro de Ciências Exactas e da Engenharia da Universidade da Madeira, Campus Universitário da Penteada, \\ 9000-390 Funchal, Portugal \\ ${ }^{\mathrm{b}}$ Department of Physical and Analytical Chemistry, University of Jaén, Campus Las Lagunillas S/N, E-23071 Jaén, Spain
}

\section{A R T I C L E I N F O}

\section{Article history:}

Received 24 July 2014

Received in revised form 29 October 2014

Accepted 30 October 2014

Available online 15 November 2014

\section{Keywords:}

Macaronesia

Tandem mass spectrometry

Flavonoid

Procyanidin

Endemic species

\begin{abstract}
A B S T R A C T
Phenolic compounds present high antioxidant activity and, therefore, health promoting effects, serving as a type of preventive medicine. Hence, research on the chemical composition of plants with potential antioxidant value is of high interest.

Forest cleaning, thinning, and pruning are beneficial activities that help maintaining healthy forests. In addition, they can provide vegetal material as source of valuable bioactive compounds that can have health promoting effects. In this work, the phenolic composition of several trees native to Madeira Archipelago (Portugal) was studied. Specifically, the leaves from Olea europaea ssp. cerasiformis, Ilex perado ssp. perado, Clethra arborea, and Heberdenia excelsa have been analyzed. The screening of the main phenolic compounds from their methanolic extracts has been carried out using high-performance liquid chromatography with electrospray ionization mass spectrometric detection (HPLC-ESI-MS ${ }^{\mathrm{n}}$ ). This is the first report on the phenolic composition of these Madeira native species, and more than 100 compounds have been detected and identified or tentatively characterized.
\end{abstract}

(C) 2014 Elsevier B.V. All rights reserved.

\section{Introduction}

Phenolic compounds, secondary metabolites synthesized by the plants, present high antioxidant activity and, therefore, important health benefits, such as protection against cancer, and cardiovascular and neurodegenerative diseases (Gouveia and Castilho, 2009; Ignat et al., 2011). Hence, many studies are being performed to characterize phenolic compounds from natural sources. For this purpose, liquid chromatography coupled to tandem mass spectrometry has proved to be a very powerful tool.

The Madeira laurel forest, Laurisilva, is a subtropical forest with a very rich bryophyte and vascular flora. It is well characterized from the botanical point of view but its chemistry remains unexplored, even though several species have been used for centuries in the preparation of folk remedies (Rivera, 1995). In this work, the most important non-lauraceae trees of the Laurisilva forest (Olea europaea ssp. cerasiformis, Ilex perado ssp. perado, Clethra arborea,

\footnotetext{
* Corresponding author. Tel.: +351 291705102.

E-mail address: castilho@uma.pt (P.C. Castilho).
}

and Heberdenia excelsa) have been selected, and their phenolic composition studied.

The olive tree (Olea europaea L., Oleaceae) is a fruit crop of high economic importance. Six subspecies of $O$. europaea have been described. Of them, ssp. cerasiformis Webb and Berth. ex Kunkel and Sunding (previously named O. europaea L. ssp. maderensis Lowe) is native to the Madeira Archipelago (Brito et al., 2008). It is locally known as "Oliveira brava" or "zambujeiro" and grows widely at altitudes between 0 and $200 \mathrm{~m}$. Unlike the 0 . europaea cultivar developed for fruit and oil production, the fruits of the endemic wild species are inedible, but infusions of its leaves are used as an antihypertensive. Although previous studies have been carried out regarding the phenolic composition of O. europaea (Fu et al., 2010; Quirantes-Piné et al., 2013; Savarese et al., 2007), this is the first study for the Madeira native species.

The genus Ilex L. (Aquifoliaceae) includes over 400 species of trees and shrubs. I. perado Aiton, a complex of four subspecies distributed in different Macaronesian archipelagos, is represented in Madeira by I. perado ssp. perado Aiton (Sosa et al., 2013). In the Ilex genus, the phenolic composition of I. paraguensis has been previously reported (Bastos et al., 2007; Dartora et al., 2011; Peres et al., 
2013), but no data are available regarding ssp. perado. It is known as Madeira Holy or "azevinho".

Clethra arborea Aiton, also known as the lily-of-the-valley-tree or "folhado", is a flowering plant in the genus Clethra. It is native to Madeira, extinct in the Canary Islands (Spain), and an invasive species in the Azores (Portugal). To our best knowledge, the phenolic composition of the Clethra genus has not been studied to date.

Heberdenia excelsa Aiton (Ardisia excelsa A.) is an uncommon species of Laurisilva, native to Madeira and to the Canary Islands, and known locally as "aderno". It is currently included in the International Union for Conservation of Nature (IUCN) Red List of Threatened Species (IUCN, 2013). The phenolic composition of plants in the Heberdenia genus has been scarcely studied to date (de Mejía et al., 2006), the report presented here being the first one for the ssp. excelsa.

Being protected as part of UNESCO natural patrimony since 2000 , every year the Laurisilva forest must be cleaned and thinned to prevent fire spread and to improve the growth of healthy trees. Presently, the felled specimens and cut branches are discarded or treated as biomass residue without further valorization. The present work is part of a prospective project that aims at finding uses for discarded material for forest valorization.

\section{Experimental}

\subsection{Chemicals and reagents}

HPLC grade acetonitrile $\left(\mathrm{CH}_{3} \mathrm{CN}\right)$ (99\%; LabScan; Dublin, Ireland), ultrapure water (Milli-Q Waters purification system; Millipore; Milford, MA, USA), and formic acid (analytical reagent grade; Sigma-Aldrich; St. Louis, MO, USA), were used in the LC-MS analysis. The methanol used for the extraction procedures, of analytical reagent grade, was obtained from Fisher (Lisbon, Portugal). Eluents LC-MS analysis were also filtered through $0.45 \mu \mathrm{m}$ Nylon membranes (Millipore; Merck; Darmstadt, Germany). Charcoal activated powder was purchased from Sigma-Aldrich.

Quercetin (>99\%) was obtained from ExtraSynthese (Lyon, France). Kaempferol (>99\%) and 5-O-caffeoylquinic acid (99\%) were purchased from Acros Organics (Geel, Belgium). 3,4O-dicaffeoylquinic acid, 3,5-O-dicaffeoylquinic acid and 4,5-Odicaffeoylquinic acid were purchased from Chengdo biopurity phytochemicals (Ltd China, Sichuan, China). Rutin (>94\%) was obtained from Sigma-Aldrich.

\subsection{Sample preparation and extraction of phenolic compounds}

Samples of Clethra arborea, Heberdenia excelsa, Ilex perado, and Olea europaea were collected in the wild in Madeira Island, in June 2012, with the help of Professor Miguel Menezes de Sequeira from the Biology Department of Madeira University. Vouchers were deposited in the Madeira Botanical Garden Herbarium collection. Specimen collection was performed at full maturity of leaves, in a protected forest area, of restricted human access and free of contamination of introduced species. The leaves were de-stemmed, lyophilized to dryness (Savant vapour trap RVT400; Thermo Scientific Inc.; Waltham, MA, USA), ground to powder, and stored at $-20^{\circ} \mathrm{C}$ until analysis.

The phenolic compounds were extracted by ultrasound-assisted extraction. Using a sonicator Bandelin Sonorex (Germany), $1 \mathrm{~g}$ of plant material was extracted with $25 \mathrm{~mL}$ of methanol (room temperature) at $35 \mathrm{~Hz}$ and $200 \mathrm{~W}$ for $60 \mathrm{~min}$. Then, chlorophylls were removed by adsorption on activated charcoal and extracts were filtered and concentrated to dryness in a rotary evaporator (Buchi Rotavapor R-114; USA) at $40^{\circ} \mathrm{C}$. The extracts were stored at $-20^{\circ} \mathrm{C}$ until use. For HPLC analysis, the extracts were dissolved in the initial
HPLC mobile phase, to obtain solutions of $5 \mathrm{mg} \mathrm{mL}^{-1}$ concentrations.

\subsection{Chromatographic conditions}

The HPLC analysis was carried out on a Dionex ultimate 3000 series instrument (Thermo Scientific Inc.) coupled to a binary pump, an autosampler and a column compartment (kept at $20^{\circ} \mathrm{C}$ ). Separation was achieved on a Phenomenex Gemini $\mathrm{C}_{18}$ column $(5 \mu \mathrm{m}$, $250 \times 3.0 \mathrm{~mm}$ i.d.) using a mobile phase composed by $\mathrm{CH}_{3} \mathrm{CN}(\mathrm{A})$ and water/formic acid $(0.1 \%, \mathrm{v} / \mathrm{v})$ at a flow rate of $0.4 \mathrm{~mL} \mathrm{~min}^{-1}$. The following gradient program was used: $20 \% \mathrm{~A}(0 \mathrm{~min}), 25 \% \mathrm{~A}$ (10 min), 25\% A (20 min), 50\% A (40 min), 100\% A (42-47 min) and $20 \%$ A (49-55 min).

For HPLC-ESI-MSn analysis, a Bruker Esquire model 6000 ion trap mass spectrometer (Bremen, Germany) with an ESI source was used. MS ${ }^{\mathrm{n}}$ analysis was performed in negative and positive mode and scan range was set at $m / z 100-1000$ with speed of $13,000 \mathrm{Da} / \mathrm{s}$. The conditions of ESI were as follows: drying and nebulizer gas $\left(\mathrm{N}_{2}\right)$ flow rate and pressure, $10 \mathrm{~mL} \mathrm{~min}^{-1}$ and $50 \mathrm{psi}$; capillary temperature, $325^{\circ} \mathrm{C}$; capillary voltage, $4.5 \mathrm{keV}$; collision gas (He) pressure and energy, $1 \times 10^{-5}$ mbar and $40 \mathrm{eV}$. The acquisition of $\mathrm{MS}^{\mathrm{n}}$ data was made with the auto $\mathrm{MS}^{\mathrm{n}}$ mode, selecting an isolation width of $4.0 \mathrm{~m} / \mathrm{z}$, and a fragmentation amplitude of $1.0 \mathrm{~V}\left(\mathrm{MS}^{\mathrm{n}}\right.$ up to $\left.\mathrm{MS}^{4}\right)$. Samples were filtered through $0.45 \mu \mathrm{m}$ PTFE membrane filters, and $10 \mu \mathrm{L}$ were injected.

\section{Results and discussion}

For the analysis of the phenolic composition by HPLC-ESI-MS ${ }^{\mathrm{n}}$ both the positive and negative ionization modes were used. Practically all the information was obtained using the negative mode, and the positive mode was mainly used for confirmation purposes and for the screening of anthocyanidins. The base peak chromatograms of the methanolic extracts of each plant are shown in Figs. 1 and 2.

In general, in the negative ionization mode $\left(\mathrm{ESI}^{-}\right) \mathrm{MS}^{1}$ spectrum, the most intense peak corresponded to the deprotonated molecular ion $[\mathrm{M}-\mathrm{H}]^{-}$, allowing $\mathrm{MS}^{\mathrm{n}}$ analysis. Losses of sugar moieties like hexosyl, deoxyhexosyl, pentosyl, rutinosyl, and glucuronyl ( -162 , $-146,-132,-308$, and $-176 \mathrm{Da}$, respectively) were observed in conjugated phenolic compounds.

Compounds were numbered by their order of elution, maintaining the same numeration in all the samples. The structures of the most relevant compounds identified are shown in Figs. 3 and 4.

\subsection{Olea europaea ssp. cerasiformis}

The results obtained in the analysis of leaves extracts from Olea europaea are shown in Table $1\left(\mathrm{ESI}^{-}\right)$. Most of the compounds, as previously reported in scientific literature for other Olea subspecies, were secoiridoids and flavonoids (Fu et al., 2010; Quirantes-Piné et al., 2013).

\subsubsection{Secoiridoids}

Olea europaea L. was rich in oleosides, which are oleaceaespecific secoiridoids usually esterified to a phenolic moiety (Quirantes-Piné et al., 2013). Oleuropein (compound 61) was the most abundant compound, which is in agreement with scientific bibliography (Altiok et al., 2008; Benavente-García et al., 2000; Briante et al., 2002; De Nino et al., 1997; Fu et al., 2010; Mylonaki et al., 2008; Pereira et al., 2007; Quirantes-Piné et al., 2013). The identification of oleuropein was based on its $[\mathrm{M}-\mathrm{H}]^{-}$at $m / z 539$, and its characteristic fragmentation pattern (Bianco et al., 2001; Fu et al., 2010), with fragment ions at $m / z$ 377, 307, and 275 . 
Table 1

Characterization of the methanolic extracts from leaves of Olea europaea ssp. cerasiformis.

\begin{tabular}{|c|c|c|c|c|c|}
\hline No & $t_{R}(\min )$ & {$[\mathrm{M}-\mathrm{H}]^{-}(m / z)$} & HPLC-ESI-MS $; m / z$ (\% base peak) & Assigned identity & Ref. \\
\hline 1 & 2.8 & 683 & $\begin{array}{l}\mathrm{MS}^{2}[683]: 342(10.3), 341(100) \\
\operatorname{MS}^{3}[683 \rightarrow 341]: 179(100), 143(10.1), 119(15), 113 \\
(26.8), 101(12.9) \\
\text { MS }^{4}[683 \rightarrow 341 \rightarrow 179]: 161(65.3), 143(50.7), 119(48.2) \\
113(61.3), 89(100)\end{array}$ & Hexose polymer & $\begin{array}{l}\text { Brudzynski and Miotto } \\
\text { (2011) }\end{array}$ \\
\hline 4 & 3.0 & 191 & $\begin{array}{l}\operatorname{MS}^{2}[191]: 173(74.7), 127(100), 111(59.1), 93 \text { (37.9), } 85 \\
(36.9) \\
\operatorname{MS}^{3}[191 \rightarrow 127]: 109(81.5), 85(100)\end{array}$ & Quinic acid & $\begin{array}{l}\text { Gouveia and Castilho } \\
\text { (2011) }\end{array}$ \\
\hline 5 & 3.1 & 731 & $\begin{array}{l}\mathrm{MS}^{2}[731]: 390(11.3), 389(100) \\
\operatorname{MS}^{3}[731 \rightarrow 389]: 345(100), 183(33.9), 165(86.6), 131 \\
(27.9), 121(38.8) \\
\mathrm{MS}^{4}[731 \rightarrow 389 \rightarrow 345]: 151(85.1), 121(82.4), 119(36), \\
113(37.5), 101(100)\end{array}$ & $\begin{array}{l}\text { Oleoside/secologanoside } \\
\text { derivative }\end{array}$ & - \\
\hline 8 & 3.9 & 317 & $\begin{array}{l}\operatorname{MS}^{2}[317]: 225(100), 165(19.5), 125(23.8) \\
\operatorname{MS}^{3}[317 \rightarrow 225]: 207(45.1), 165(100), 125(80.4), 95 \\
(84.4), 81(43.3)\end{array}$ & Unknown & - \\
\hline 9 & 4.1 & 601 & $\begin{array}{l}\mathrm{MS}^{2}[601]: 404(17.5), 403(100), 223(11.9), 179(7.5) \\
\mathrm{MS}^{3}[601 \rightarrow 403]: 371(34.3), 333(14.2), 223(100), 179 \\
(29.4), 121(21.2) \\
\mathrm{MS}^{4}[601 \rightarrow 403 \rightarrow 223]: 161(2.1), 121(100), 101(17.5)\end{array}$ & $\begin{array}{l}\text { Elenolic acid glucoside } \\
\text { derivative }\end{array}$ & Eyles et al. (2007) \\
\hline 12 & 4.3 & 389 & $\begin{array}{l}\mathrm{MS}^{2}[389]: 345(100), 227(3.1), 209(47.5), 183(15.2), 165 \\
(86.1), 121(32.1) \\
\mathrm{MS}^{3}[389 \rightarrow 345]: 183(59.7), 179(32.5), 165(100), 163 \\
(50.6), 161(16.3) \\
\operatorname{MS}^{4}[389 \rightarrow 345 \rightarrow 165]: 121(100)\end{array}$ & Oleoside/secologanoside & $\begin{array}{l}\text { Cardoso et al. (2005); } \\
\text { Fu et al. (2010) }\end{array}$ \\
\hline 13 & 4.3 & 593 & $\begin{array}{l}\mathrm{MS}^{2}[593]: 503(29.2), 474(24), 473(100), 383(32.4), 353 \\
(88.3) \\
\mathrm{MS}^{3}[593 \rightarrow 473]: 383(20.1), 354(16.4), 353(100) \\
\mathrm{MS}^{3}[593 \rightarrow 473 \rightarrow 353]: 326(10.1), 325(100), 298(21.6), \\
297(80.8)\end{array}$ & $\begin{array}{l}\text { Vicenin-2 } \\
\text { (apigenin-6,8-di-C-glucoside) }\end{array}$ & $\begin{array}{l}\text { Barreca et al. (2011); } \\
\text { Truchado et al. (2011) }\end{array}$ \\
\hline 14 & 4.4 & 447 & $\begin{array}{l}\mathrm{MS}^{2}[447]: 315(100), 163(60.4), 153(51.1), 152(78), 151 \\
(31.5) \\
\text { MS }^{3}[447 \rightarrow 315]: 163(12.2), 153(100), 135(12.8), 109 \\
(24.6), 108(73.3)\end{array}$ & $\begin{array}{l}\text { 3,5-dihydroxybenzoicacid- } \\
\text { glucopylxyloside }\end{array}$ & Han et al. (2008) \\
\hline 19 & 5 & 437 & $\begin{array}{l}\operatorname{MS}^{2}[437]: 402(13.8), 401(100) \\
\operatorname{MS}^{3}[437 \rightarrow 401]: 270(17), 269(100), 233(16.3), 161(96.2) \\
\operatorname{MS}^{4}[437 \rightarrow 401 \rightarrow 269]: 161(100), 159(15.6), 126(19)\end{array}$ & Unknown & - \\
\hline 20 & 5.2 & 377 & $\begin{array}{l}\operatorname{MS}^{2}[377]: 153(29.9), 197(100) \\
\operatorname{MS}^{3}[377 \rightarrow 197]: 153(100)\end{array}$ & Oleuropein aglycone derivative & $\begin{array}{l}\text { Fu et al. (2010); Jemai } \\
\text { et al. (2009) }\end{array}$ \\
\hline 24 & 5.8 & 807 & $\operatorname{MS}^{2}[807]: 404$ (13.2), 403 (100), 371 (14.5) & Elenolic acid glucoside & Fu et al. (2010) \\
\hline
\end{tabular}

MS $^{3}$ [807 $\rightarrow$ 403]: 371 (100), 333 (7.4), 223 (58.1), 179

(28.4), $121(18.6)$

$\mathrm{MS}^{4}[807 \rightarrow 403 \rightarrow 371]: 166(17.3), 165(19.5), 121(100)$

$26 \quad 739$

$\mathrm{MS}^{2}$ [739]: 594 (20.7), 593 (100), 286 (14.8), 285 (68.6)

$\mathrm{MS}^{3}$ [739 $\rightarrow$ 593]: 447 (20.5), 285 (100)

MS $^{4}[739 \rightarrow 593 \rightarrow 285]: 267$ (86.8), 257 (55.8), 197 (30.5),

151 (100)

$29 \quad 6.9 \quad 525$

$30 \quad 693$

$33 \quad 7.2 \quad 609$

$37 \quad 7.5 \quad 623$

$\begin{array}{lll}40 & 8.1 & 447\end{array}$

$44 \quad 8.6 \quad 701$

$\begin{array}{lll}47 & 9.5 & 701\end{array}$
MS $^{2}$ [525]: 481 (100), 389 (60.2), 345 (35.2), 195 (70), 165 (57.9)

MS $^{3}[525 \rightarrow 481$ ]: 345 (54.5), 319 (17.6), 301 (68.6), 255

(100), 195 (44.5), 139 (59.5)

$\mathrm{MS}^{2}$ [593]: 286 (13.4), 285 (100)

$\mathrm{MS}^{3}[593 \rightarrow 285$ ]: 243 (76.4), 241 (56.7), 211 (100), 199

(78.8), 175 (73.8), 151 (19.8), 149 (91.4)

$\mathrm{MS}^{4}[593 \rightarrow 285 \rightarrow 149]: 105(100)$

$\mathrm{MS}^{2}$ [609]: 302 (11.5), 301 (100), 300 (25.3)

$\mathrm{MS}^{3}[609 \rightarrow 301$ ]: 271 (22), 255 (30.9), 179 (94.2), 151

(100), 107 (10.5)

$\mathrm{MS}^{4}[609 \rightarrow 301 \rightarrow 151]: 169$ (54.1), $107(100)$

$\mathrm{MS}^{2}[623]: 462$ (17.6), 461 (100)

$\mathrm{MS}^{3}[623 \rightarrow 461]: 315$ (34.2), 161 (10.3), 135 (100)

$\mathrm{MS}^{2}$ [447]: 286 (19.1), 285 (100)

$\mathrm{MS}^{3}[447 \rightarrow 285$ ]: 243 (52.5), 241 (100), 217 (60.6), 175

(74.9), 151 (91.6)

$\mathrm{MS}^{4}[447 \rightarrow 285 \rightarrow 241]: 213$ (47.7), 201 (51.7), 200 (57),

199 (75.7), 197 (100)

$\mathrm{MS}^{2}$ [701]: 540 (29.6), 539 (100), 307 (10.5)

$\mathrm{MS}^{3}[701 \rightarrow 539$ ]: 377 (13.3), 371 (11.8), 307 (100), 275

(87.5)

$\mathrm{MS}^{4}[701 \rightarrow 539 \rightarrow$ 307]: 275 (100), 139 (23.4)

MS $^{2}$ [701]: 540 (21.8), 539 (100), 377 (13.4), 307 (18.7),

275 (16.2)

$\mathrm{MS}^{3}$ [701 $\rightarrow$ 539]: 469 (16.7), 377 (16.8), 327 (12), 307

(100), 275 (59.2)

$\mathrm{MS}^{4}[701 \rightarrow 539 \rightarrow$ 307]: 276 (16.4), 275 (100), 139 (27.7)

Kaempferol-rhamnoside-hexoside-rhahriosidel. (2004)

Demethyloleuropein

Savarese et al. (2007)

Luteolin 7-rutinoside

Rutin

Verbascoside

Luteolin glucoside

Oleuropein diglucoside isomer

Oleuropein diglucoside isome
Cardoso et al. (2005): Quirantes-Piné et al. (2013)

a 
Table 1 (Continued)

\begin{tabular}{|c|c|c|c|c|c|}
\hline No & $t_{R}(\min )$ & {$[\mathrm{M}-\mathrm{H}]^{-}(m / z)$} & HPLC-ESI-MS ${ }^{n} ; m / z$ (\% base peak) & Assigned identity & Ref. \\
\hline 51 & 10.3 & 551 & $\begin{array}{l}\mathrm{MS}^{2}[551]: 508(23.3), 507(100), 389(19.5), 341(14.4), \\
281(27.1) \\
\text { MS }^{3}[551 \rightarrow 507]: 393(16.1), 345(17.7), 323(11.5), 179 \\
(18.2), 161(100) \\
\operatorname{MS}^{4}[551 \rightarrow 507 \rightarrow 161]: 133(100)\end{array}$ & $6^{\prime}$ - $\beta$-hexopyranosyloleoside & $\begin{array}{l}\text { Cardoso et al. (2005); } \\
\text { Damak et al. (2008) }\end{array}$ \\
\hline 55 & 11.3 & 447 & $\begin{array}{l}\operatorname{MS}^{2}[447]: 302(14.6), 301(100), 300(33.2) \\
\operatorname{MS}^{3}[447 \rightarrow 301]: 271(16.9), 255(15.7), 179(94.4), 151 \\
(100) \\
\operatorname{MS}^{4}[447 \rightarrow 301 \rightarrow 151]: 108(22.5), 107(100), 83(26.1)\end{array}$ & Quercetin-O-rhamnoside & - \\
\hline 57 & 12.9 & 569 & $\begin{array}{l}\mathrm{MS}^{2}[569]: 538(19.1), 537(100), 403(44.4), 223(17.5) \\
\mathrm{MS}^{3}[569 \rightarrow 537]: 403(12.5), 357(27.5), 305(12.2), 223 \\
(82.4), 151(100) \\
\mathrm{MS}^{4}[569 \rightarrow 537 \rightarrow 151]: 123(100) ; \\
\operatorname{MS}^{4}[569 \rightarrow 537 \rightarrow 223]: 121(100)\end{array}$ & Unknown & - \\
\hline 59 & 13.3 & 447 & $\begin{array}{l}\mathrm{MS}^{2}[447]: 286(16.8), 285(100) \\
\mathrm{MS}^{3}[447 \rightarrow 285]: 243(18.7), 241 \text { (56.5), } 217(17.2), 199 \\
(73.9), 175(100)\end{array}$ & Luteolin glucoside & $\begin{array}{l}\text { Cardoso et al. (2005); } \\
\text { Fu et al. (2010); } \\
\text { Quirantes-Piné et al. } \\
\text { (2013) }\end{array}$ \\
\hline 61 & 13.7 & 539 & $\begin{array}{l}\mathrm{MS}^{2}[539]: 377(56.1), 308(12), 307(100), 275(93.7) \\
\mathrm{MS}^{3}[539 \rightarrow 307]: 276(14.8), 275(100), 139(27.5), 111 \\
(13.8) \\
\mathrm{MS}^{4}[539 \rightarrow 307 \rightarrow 275]: 149(59), 139(100), 121(16.3), \\
113(89), 111(58.4)\end{array}$ & Oleuropein & $\begin{array}{l}\text { Bianco et al. (2001); Fu } \\
\text { et al. (2010) }\end{array}$ \\
\hline 64 & 15 & 539 & $\begin{array}{l}\mathrm{MS}^{2}[539]: 377(36.8), 345(22.6), 327(17.9), 307(100), \\
275(98) \\
\text { MS }^{3}[539 \rightarrow 307]: 275(100), 139(25.9) \\
\text { MS }^{4}[539 \rightarrow 307 \rightarrow 275]: 149(70.4), 139(70), 113(100), \\
111(31.7)\end{array}$ & Oleuropein isomer & $\begin{array}{l}\text { Fu et al. (2010); } \\
\text { Quirantes-Piné et al. } \\
\text { (2013) }\end{array}$ \\
\hline 68 & 15.9 & 539 & $\begin{array}{l}\mathrm{MS}^{2}[539]: 403(16.8), 377(16.8), 307(88.2), 276(21.6), \\
275(100) \\
\text { MS }^{3}[539 \rightarrow 275]: 139(100), 123(11.9), 113(23.1), 111 \\
(12.4), 95(57.4) \\
\operatorname{MS}^{4}[539 \rightarrow 307 \rightarrow 275]: 139(100), 149(46.4), 113(94.7), \\
111(58.3), 85(16.1) \\
\text { MS }^{3}[539 \rightarrow 307]: 275(100), 139(15.3), 111(10.4) \\
\text { MS }^{4}[539 \rightarrow 275 \rightarrow 139]: 111(17.5), 96(11.3), 95(100)\end{array}$ & Oleuroside & $\begin{array}{l}\text { Fu et al. (2010); } \\
\text { Quirantes-Piné et al. } \\
\text { (2013) }\end{array}$ \\
\hline 73 & 18.6 & 601 & 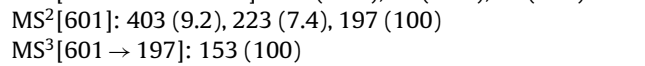 & Unknown & - \\
\hline 74 & 19.9 & 523 & $\begin{array}{l}\operatorname{MS}^{2}[523]: 362(13.9), 361(89.4), 291(100), 260(11.7) \\
259(48.4) \\
\operatorname{MS}^{3}[523 \rightarrow 291]: 171(12.5), 143(12.8), 139(65.2), 127 \\
(14.4), 111(100) \\
\operatorname{MS}^{3}[523 \rightarrow 361]: 291(100), 259(59.8) \\
\operatorname{MS}^{4}[523 \rightarrow 361 \rightarrow 291]: 259(16.2), 171(25.9), 143(11.5), \\
139(46.1), 111(100)\end{array}$ & Ligstroside & $\begin{array}{l}\text { Briante et al. (2002); } \\
\text { Laguerre et al. (2009) }\end{array}$ \\
\hline 79 & 23.8 & 557 & $\begin{array}{l}\mathrm{MS}^{2}[557]: 514(16.3), 513(100), 389(1.8), 345(35.4), 199 \\
(23.2), 185(39) \\
\mathrm{MS}^{3}[557 \rightarrow 513]: 345(100), 227(50.8), 209(52.4), 185 \\
(86.6), 183(61.5) \\
\text { MS }^{4}[557 \rightarrow 513 \rightarrow 345]: 183(100), 165(53.1), 121(42.2) \\
119(40.8), 101(46)\end{array}$ & $\begin{array}{l}\text { Oleoside/secologanoside } \\
\text { derivative }\end{array}$ & - \\
\hline 80 & 25.2 & 301 & $\begin{array}{l}\mathrm{MS}^{2}[301]: 299(13.8), 273(13.5), 179(93.3), 151(100) \\
\operatorname{MS}^{3}[301 \rightarrow 151]: 169(45.4), 107(100)\end{array}$ & Quercetin & a \\
\hline 81 & 26.8 & 877 & 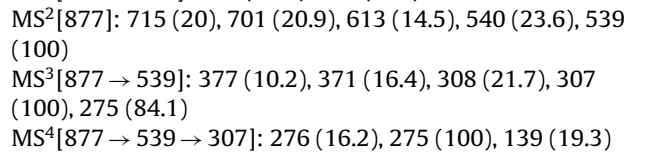 & Oleuropein derivative & - \\
\hline 82 & 27.3 & 925 & $\begin{array}{l}\mathrm{MS}^{2}[925]: 540(16.7), 539(100), 377(12.9), 307(11.7) \\
\mathrm{MS}^{3}[925 \rightarrow 539]: 377(50.7), 307(100), 276(11), 275(77.1) \\
\mathrm{MS}^{4}[925 \rightarrow 539 \rightarrow 307]: 275(100), 139(22.1), 111(10.9)\end{array}$ & Oleuropein derivative & - \\
\hline 83 & 27.7 & 539 & $\begin{array}{l}\mathrm{MS}^{2}[539]: 377(58), 308(12.7), 307(93.2), 276(11.8), 275 \\
(100) \\
\mathrm{MS}^{3}[539 \rightarrow 275]: 139(100), 113(15.9), 111(13.5), 95 \\
(48.7) \\
\mathrm{MS}^{4}[539 \rightarrow 275 \rightarrow 139]: 111(13.6), 96(10.4), 95(100) \\
\mathrm{MS}^{3}[539 \rightarrow 307]: 276(15.4), 275(100), 139(21.6) \\
\mathrm{MS}^{4}[539 \rightarrow 307 \rightarrow 275]: 149(69.4), 139(39.2), 135(20), \\
113(100), 111(16.1)\end{array}$ & Oleuropein isomer & - \\
\hline 86 & 29 & 539 & $\begin{array}{l}\mathrm{MS}^{2}[539]: 437(11.9), 377(58), 307(100), 276(13.7), 275 \\
(87.5) \\
\text { MS }^{3}[539 \rightarrow 275]: 139(100), 113(21.4), 111(46.5), 95 \\
(51.7) \\
\operatorname{MS}^{4}[539 \rightarrow 275 \rightarrow 139]: 111(81.4), 96(100) \\
\operatorname{MS}^{3}[539 \rightarrow 307]: 275(100), 139(13.6) \\
\operatorname{MS}^{4}[539 \rightarrow 307 \rightarrow 275]: 149(45.6), 139(100), 113(63), \\
111(98.9)\end{array}$ & Oleuropein isomer & - \\
\hline
\end{tabular}


Table 1 (Continued)

\begin{tabular}{|c|c|c|c|c|c|}
\hline No & $t_{R}(\min )$ & {$[\mathrm{M}-\mathrm{H}]^{-}(m / z)$} & HPLC-ESI-MSn $; m / z$ (\% base peak) & Assigned identity & Ref. \\
\hline 90 & 31.8 & 615 & $\begin{array}{l}\mathrm{MS}^{2}[615]: 286(14.4), 285(100) \\
\mathrm{MS}^{3}[615 \rightarrow 285]: 243(53.9), 241 \text { (61.3), } 200(58.4), 199 \\
(73.6), 175(100)\end{array}$ & Luteolin derivative & - \\
\hline 102 & 39.1 & 587 & $\begin{array}{l}\operatorname{MS}^{2}[587]: 300(12), 299(100) \\
\operatorname{MS}^{3}[587 \rightarrow 299]: 271(100) \\
\operatorname{MS}^{4}[587 \rightarrow 299 \rightarrow 271]: 243(74), 227(84.9), 201(32.1) \\
199(100), 157(26.6)\end{array}$ & Unknown & - \\
\hline
\end{tabular}

a Compared with standard compound.

Compounds $64,68,83$, and 86 presented the same $[\mathrm{M}-\mathrm{H}]^{-}$ ion that oleuropein did, and similar fragmentation patterns. Compound 68 was identified as oleuroside, oleuropein secoxyloganin analog (Obied et al., 2007). The presence of oleuroside and another oleuropein isomer (compound 64) has been previously described (Fu et al., 2010; Quirantes-Piné et al., 2013). However, to our best knowledge, compounds $\mathbf{8 3}$ and $\mathbf{8 6}$, also oleuropein isomers considering MS/MS data, are here reported for the first time.

Compound 20 displayed an $[\mathrm{M}-\mathrm{H}]^{-}$ion at $\mathrm{m} / \mathrm{z} 377$, with MS $^{2}$ fragment ions at $m / z 197$ and 153, and was identified as an oleuropein aglycone derivative (Fu et al., 2010; Jemai et al., 2009).

Compounds 44 and 47 displayed deprotonated molecules at $m / z$ 701. Their $\mathrm{MS}^{2}$ fragmentation profile showed base peak ion at $m / z 539$, formed by the loss of $162 \mathrm{Da}$ (hexoside residue). The $\mathrm{MS}^{3}[701 \rightarrow 539]$ fragmentation produced main fragment ions at $m / z$ 377, 307 and 275, typical from oleuropein. These compounds were characterized as oleuropein diglucoside isomers (Fu et al., 2010; Quirantes-Piné et al., 2013). Compounds 81 and 82 were tentatively characterized as oleuropein derivatives considering the fragment ion at $m / z 539$, which displayed the fragmentation pattern of oleuropein.

Compound 12 exhibited $[\mathrm{M}-\mathrm{H}]^{-}$at $m / z$ 389, with $\mathrm{MS}^{2}$ fragment ions at $m / z 345$ (base peak), 227, 209, and 183. The fragment ion at $\mathrm{m} / z 345$ was formed by the neutral loss of $44 \mathrm{Da}\left(\mathrm{CO}_{2}\right.$ molecule from a carboxylic group). The fragment ions at $\mathrm{m} / \mathrm{z} 227$ and 209 corresponded to the loss of hexoside and hexoside $+\mathrm{H}_{2} \mathrm{O}$, respectively. This fragmentation pattern was consistent with oleoside or secologanoside (Cardoso et al., 2005; Fu et al., 2010). Compounds 5 and 79 presented fragment ions at $m / z 389$ and 345, with fragmentation patterns similar to oleoside/secologanoside, so they were tentatively characterized as derivatives.
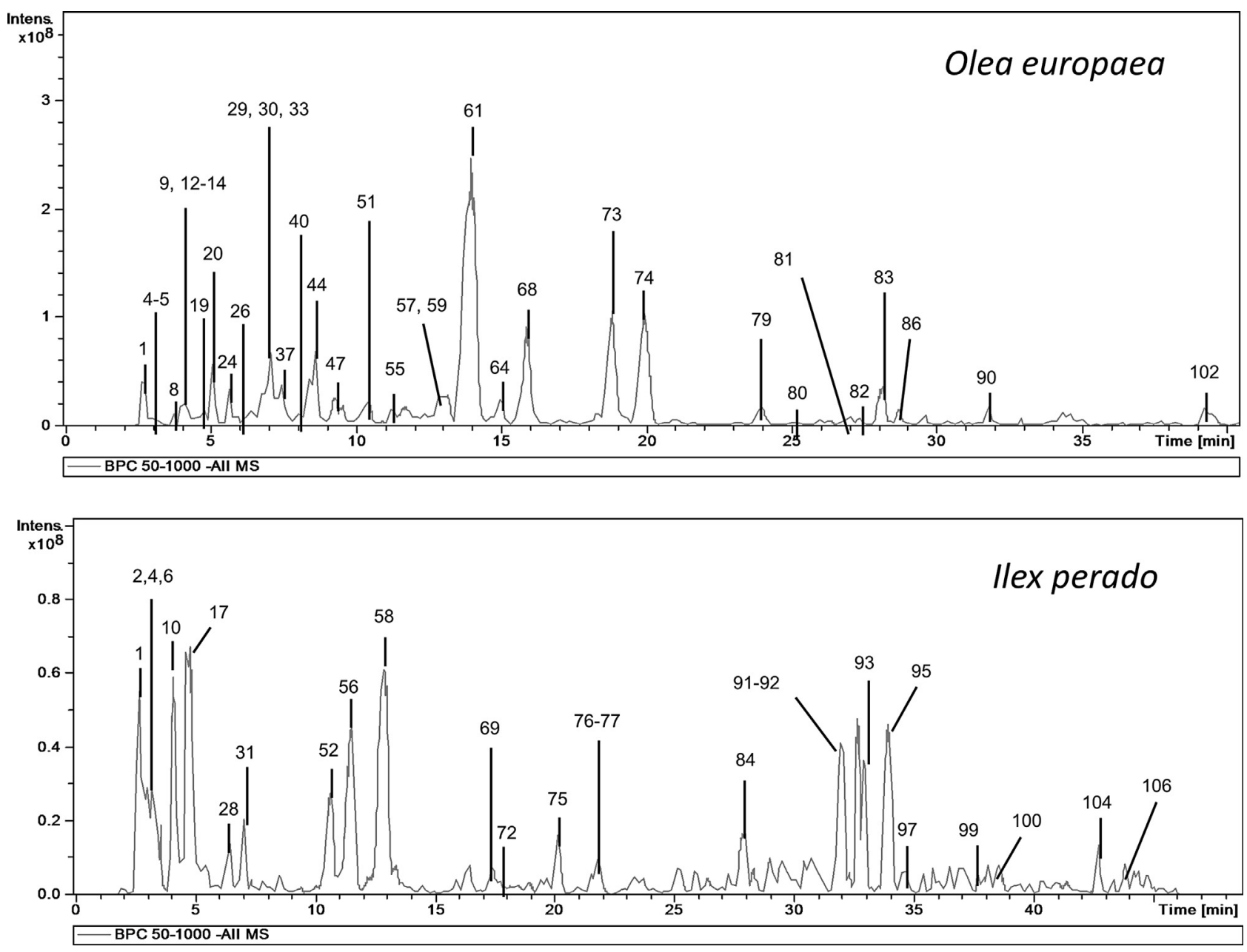

Fig. 1. HPLC-ESI/MS ${ }^{n}$ base peak chromatograms (BPC) of the methanolic extracts from Olea europaea and Ilex perado. 

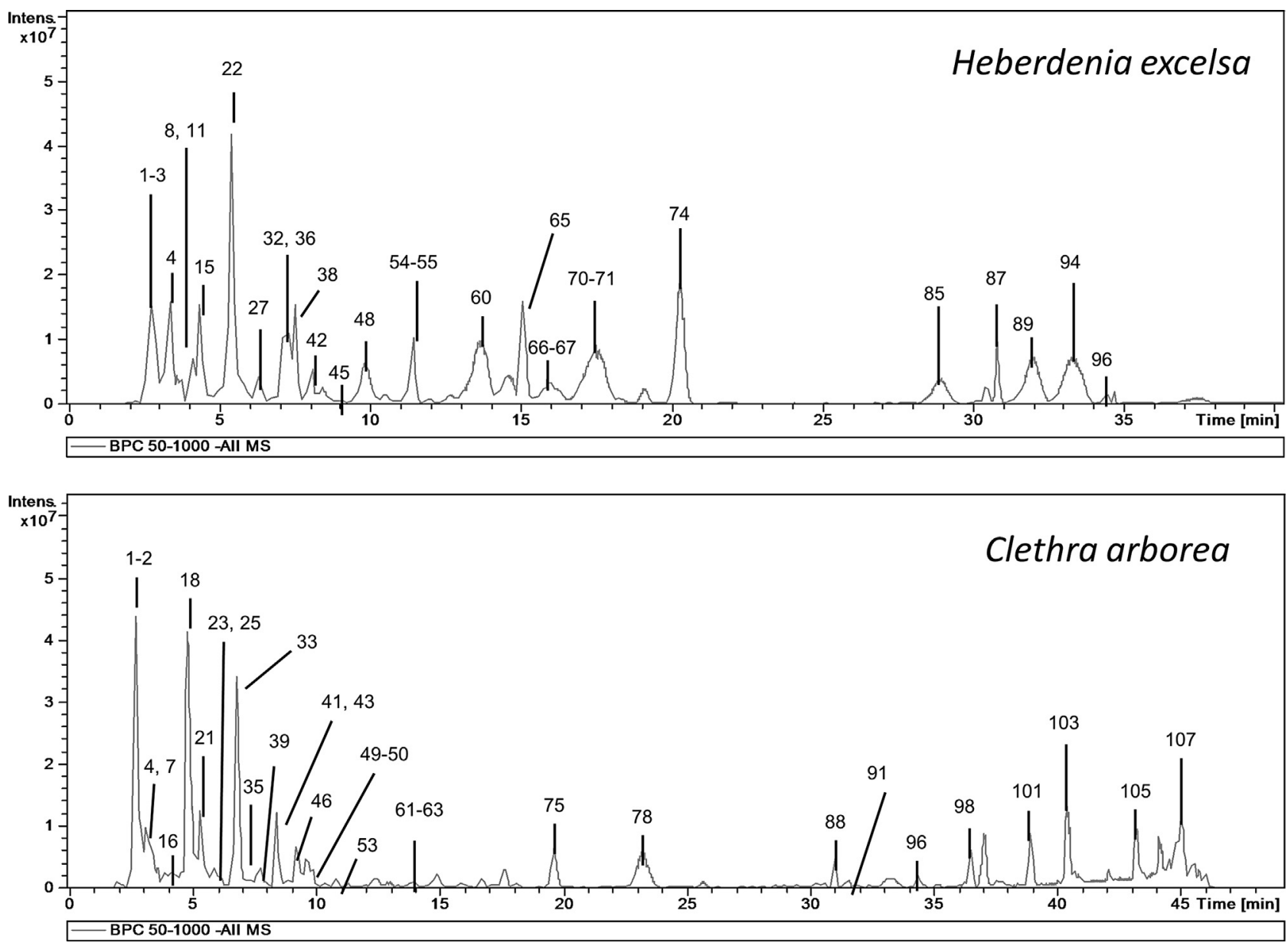

Fig. 2. HPLC-ESI/MS ${ }^{n}$ base peak chromatograms (BPC) of the methanolic extracts from Heberdenia excelsa and Clethra arborea.

Compound 24 showed $[\mathrm{M}-\mathrm{H}]^{-}$at $m / z$ 807, and an intense peak at $m / z 403$ in $M S^{1}$. Its MS ${ }^{2}$ base peakion was present at $m / z 403$, indicating that the compound was a dimer. The $\mathrm{MS}^{3}$ spectrum exhibited fragment ions at $m / z 371$ (base peak), 223, and 179. This fragmentation pattern was previously reported for elenolic acid glucoside (Fu et al., 2010). Compound 9, with $[\mathrm{M}-\mathrm{H}]^{-}$at $m / z$ 601, exhibited a base peak ion at $m / z 403$, that presented the same fragmentation pattern than elenolic acid glucoside, so it was characterized as a derivative (Eyles et al., 2007). Another oleoside derivative was compound 51, which was identified as $6^{\prime}$ - $\beta$-hexopyranosyloleoside (Cardoso et al., 2005; Damak et al., 2008).

Compound 29 was identified as demethyloleuropein, with $[\mathrm{M}-\mathrm{H}]^{-}$at $\mathrm{m} / \mathrm{z} 525$ and $\mathrm{MS}^{2}$ fragment ions at $\mathrm{m} / \mathrm{z} 389$ and 319 (Savarese et al., 2007). Compound 74 was a deoxy analog of oleuropein, ligstroside, also previously reported in olive leaves (Briante et al., 2002; Laguerre et al., 2009).

\subsubsection{Flavonoids}

Rutin (compound 33) and quercetin (compound 80) were detected as aglycones and characterized using analytical standards. In addition, several derivatives of apigenin, kaempferol, and luteolin (compounds 13, 26, 30, 40, 59, 91) were observed.

Compound 13 presented an $[\mathrm{M}-\mathrm{H}]^{-}$ion at $m / z$ 593, with $\mathrm{MS}^{2}$ fragment ions at $m / z 473$ (base peak), 503, 383 and 353. It was identified as vicenin-2, an apigenin-6,8-di-C-glucoside (Barreca et al., 2011; Truchado et al., 2011). To our knowledge, this compound has not been previously reported in Olea europaea.

Compound 26 presented deprotonated molecular ion at $\mathrm{m} / \mathrm{z}$ 739 , and suffered neutral losses of $146 \mathrm{Da}$ (rhamnoside) and $162 \mathrm{Da}$ (hexoside) in its $\mathrm{MS}^{\mathrm{n}}$ fragmentation to produce the fragment ions $[\mathrm{M}-\mathrm{H} \text {-rhamnose }]^{-}$at $\mathrm{m} / \mathrm{z} 593$, [M-H-rhamnose-rhamnose ${ }^{-}$at $m / z$ 447, and [M-H-rhamnose-hexose-rhamnose $]^{-}$at $m / z 285$. The fragment ion at $m / z 285$ corresponded to the aglycone kaempferol (main fragment ion at $\mathrm{m} / \mathrm{z} 151$, and absence of fragment ions at $\mathrm{m} / \mathrm{z}$ 243 and 241, which are typical from luteolin). This compound was identified as kaempferol-rhamnoside-hexoside-rhamnoside (Del Rio et al., 2004).

Compounds 30, 40, and 59, exhibited $[\mathrm{M}-\mathrm{H}]^{-}$ions at $m / z 593$, 447 , and 447, respectively, originating in all cases a base peak ion at $\mathrm{m} / \mathrm{z} 285$ (luteolin) in the MS ${ }^{2}$ spectrum. Hence, these compounds were tentatively identified as luteolin-7-rutinoside, and luteolin glucoside isomers, based on bibliographic data previously reported in Olea europaea (Cardoso et al., 2005; Fu et al., 2010; Quirantes-Piné et al., 2013). Compound $\mathbf{9 0}$ was tentatively identified as a luteolin derivative.

Compound $\mathbf{5 5}$ was characterized as quercetin rhamnoside. It presented an $[\mathrm{M}-\mathrm{H}]^{-}$ion at $m / z 447$, and an $\mathrm{MS}^{2}$ fragment ion at $\mathrm{m} / \mathrm{z} 301$ by the loss of a rhamnoside moiety (146 Da). The fragment ion at $m / z 301$ corresponded to quercetin (characteristic fragment ion at $m / z 151)$.

\subsubsection{Phenolic acids}

Compound 14 was characterized as a benzoic acid glucoside, 3-5,dihydroxy-benzoicacid-glucopylxyloside, based on its $[\mathrm{M}-\mathrm{H}]^{-}$ ion at $m / z$ 447, and $\mathrm{MS}^{2}$ and $\mathrm{MS}^{3}$ base peaks at $\mathrm{m} / \mathrm{z} 315$ and 153 , respectively (Han et al., 2008).

Compound 37 displayed an $[\mathrm{M}-\mathrm{H}]^{-}$ion at $m / z$ 623, and fragment ions at $m / z 461,315,161$ and 135 . The fragment ions at $m / z$ 
<smiles>O=C(O)C1(O)CC(O)C(O)C(O)C1</smiles>

Quinic acid<smiles>O=C(O)/C=C/c1ccc(O)c(O)c1</smiles>

Caffeic acid<smiles>O=C(O)/C=C/c1ccc(O)cc1</smiles>

p-Coumaric acid<smiles>O=C(/C=C/c1ccc(O)c(O)c1)OC1CCC(O)(C(=O)O)CC(O)C1O</smiles>

5-O-caffeoylquinic acid<smiles>O=C(/C=C/c1ccc(O)c(O)c1)O[C@@H](Cc1ccc(O)c(O)c1)C(=O)O</smiles>

Rosmarinic acid<smiles>O=C(/C=C/c1ccc(O)c(O)c1)O[C@H](C(=O)O)[C@@H](O)C(=O)O</smiles>

Caftaric acid<smiles>[R7]c1cc([C@H]2CC(=O)c3c(O)cc(O)cc3O2)ccc1O</smiles>

Eriodyctiol

$\mathrm{R}_{1}=\mathrm{OH}$

Naringenin

$\mathrm{R}_{1}=\mathrm{H}$<smiles>[R]c1cc(-c2oc3cc(O)cc(O)c3c(=O)c2[R])cc([R])c1[R]</smiles>

Kaempferol

Luteolin Mearnsetin

Myricetin

Rutin

Quercetin
$\mathrm{R}_{1}=\mathrm{H} ; \mathrm{R}_{2}=\mathrm{OH} ; \mathrm{R}_{3}=\mathrm{H} ; \mathrm{R}_{4}=\mathrm{OH}$

$\mathrm{R}_{1}=\mathrm{OH} ; \mathrm{R}_{2}=\mathrm{OH} ; \mathrm{R}_{3}=\mathrm{H} ; \mathrm{R}_{4}=\mathrm{H}$

$\mathrm{R}_{1}=\mathrm{OH} ; \mathrm{R}_{2}=\mathrm{OCH}_{3} ; \mathrm{R}_{3}=\mathrm{OH} ; \mathrm{R}_{4}=\mathrm{OH}$

$\mathrm{R}_{1}=\mathrm{OH} ; \mathrm{R}_{2}=\mathrm{OH} ; \mathrm{R}_{3}=\mathrm{OH} ; \mathrm{R}_{4}=\mathrm{OH}$

$\mathrm{R}_{1}=\mathrm{H} ; \mathrm{R}_{2}=\mathrm{OH} ; \mathrm{R}_{3}=\mathrm{OH} ; \mathrm{R}_{4}=\mathrm{O}-$ Rutinose

$\mathrm{R}_{1}=\mathrm{OH} ; \mathrm{R}_{2}=\mathrm{OH} ; \mathrm{R}_{3}=\mathrm{H} ; \mathrm{R}_{4}=\mathrm{OH}$

Fig. 3. Chemical structures of phenolic acids, flavonoid aglycones, and substitution groups found in the analyzed leave extracts.

461 and 315 were caused by the sequential neutral losses of hexoside (162 Da) and rhamnoside (146 Da). This fragmentation pattern is typical from verbascoside (Cardoso et al., 2005; Fu et al., 2010).

\subsubsection{Other compounds}

Compound 4 exhibited an $[\mathrm{M}-\mathrm{H}]^{-}$ion at $\mathrm{m} / \mathrm{z}$ 191. Its $\mathrm{MS}^{2}$ fragmentation yielded a $\left[\mathrm{M}-\mathrm{H}-\mathrm{CO}-2 \mathrm{H}_{2} \mathrm{O}\right]^{-}$ion at $m / z 127$ and a $\left[\mathrm{M}-\mathrm{H}-\mathrm{H}_{2} \mathrm{O}\right]^{-}$ion at $m / z$ 173. It was identified as quinic acid
(Gouveia and Castilho, 2011). There were several compounds that could not be identified and were just stated as unknowns in Table 1.

\subsection{Ilex perado}

To our best knowledge, the determination of phenolic compounds in I. perado has not been previously reported. However, there are some available data in scientific bibliography regarding 
Table 2

Characterization of the methanolic extracts from leaves of Ilex perado ssp. perado.

\begin{tabular}{|c|c|c|c|c|c|}
\hline No & $t_{R}(\min )$ & {$[\mathrm{M}-\mathrm{H}]^{-}(m / z)$} & HPLC-ESI-MS ${ }^{n} ; m / z$ (\% base peak) & Assigned identity & Ref. \\
\hline 1 & 2.7 & 683 & $\begin{array}{l}\text { MS }^{2}[683]: 341(100) \\
\text { MS }^{3}[683 \rightarrow 341]: 179(100), 161(12.5), 143(20.8), 131(14.5) \\
119(15.5), 113(27.8) \\
\text { MS }^{4}[683 \rightarrow 341 \rightarrow 179]: 161(100), 113(99.6), 101(31.2), 89 \\
(69.9), 87(31.7)\end{array}$ & Hexose polymer & $\begin{array}{l}\text { Brudzynski and } \\
\text { Miotto (2011) }\end{array}$ \\
\hline 2 & 2.8 & 533 & $\begin{array}{l}\operatorname{MS}^{2}[533]: 192(4.7), 191(100) \\
\operatorname{MS}^{3}[533 \rightarrow 191]: 173(55.2), 171(40.7), 127(100), 109(21.5) \\
85(42) \\
\text { MS }^{4}[533 \rightarrow 191 \rightarrow 127]: 109(100), 85(68.5)\end{array}$ & Quinic acid derivative & - \\
\hline 4 & 3.0 & 191 & $\begin{array}{l}\text { MS }^{2}[191]: 173(100), 127(99), 111(63.1), 93(47), 87(31.9) \\
\text { MS }^{3}[191 \rightarrow 173]: 127(90), 111(100), 109(41.9), 94(31), 93 \\
(80.5) \\
\text { MS }^{3}[191 \rightarrow 127]: 109(43), 85(100), 81(26.2)\end{array}$ & Quinic acid & $\begin{array}{l}\text { Gouveia and } \\
\text { Castilho (2011) }\end{array}$ \\
\hline 6 & 3.1 & 353 & $\begin{array}{l}\text { MS }^{2}[353]: 191(100), 179(33.9), 173(11) \\
\text { MS }^{3}[353 \rightarrow 191]: 173(93.8), 127(100), 111(112.3), 93(41.8), \\
85(40.2) \\
\text { MS }^{4}[353 \rightarrow 191 \rightarrow 127]: 110(62.7), 109(100), 85(65)\end{array}$ & Caffeoylquinic acid & - \\
\hline 10 & 4.2 & 353 & $\begin{array}{l}\text { MS }^{2}[353]: 191(100), 179(35.4), 135(14.7) \\
\text { MS }^{3}[353 \rightarrow 191]: 173(39.5), 127(100), 111(45.4), 93(83.1), \\
85(62.5) \\
\text { MS }^{4}[353 \rightarrow 191 \rightarrow 127]: 110(32.6), 109(97.6), 85(100)\end{array}$ & $\begin{array}{l}\text { 3-O-Caffeoylquinic } \\
\text { acid }\end{array}$ & $\begin{array}{l}\text { Clifford et al. } \\
(2003)\end{array}$ \\
\hline 17 & 4.7 & 707 & $\begin{array}{l}\text { MS }^{2}[707]: 354(10.2), 353(100) \\
\text { MS }^{3}[707 \rightarrow 353]: 191(100) \\
\text { MS }^{4}[707 \rightarrow 353 \rightarrow 191]: 173(29.8), 127(100), 111(26.4), 85 \\
(30.6)\end{array}$ & $\begin{array}{l}\text { 5-O-Caffeoylquinic } \\
\text { acid }\end{array}$ & $\begin{array}{l}\text { Clifford et al. } \\
(2003)^{a}\end{array}$ \\
\hline 28 & 6.4 & 337 & $\begin{array}{l}\operatorname{MS}^{2}[337]: 191(24.3), 173(100), 163(8.3) \\
\text { MS }^{3}[337 \rightarrow 173]: 155(12.5), 111(57.1), 93(100), 81(12.1), 71 \\
(12.4) \\
\text { MS }^{3}[337 \rightarrow 191]: 173(46.1), 127(100), 111(17.9), 93(24), 85 \\
(28.5)\end{array}$ & $\begin{array}{l}\text { 4-p-Coumaroylquinic } \\
\text { acid }\end{array}$ & $\begin{array}{l}\text { Clifford et al. } \\
\text { (2006a) }\end{array}$ \\
\hline 31 & 7.1 & 551 & $\begin{array}{l}\operatorname{MS}^{2}[551]: 506(30.5), 505(100) \\
\operatorname{MS}^{3}[551 \rightarrow 505]: 374(20), 373(100), 191(12.1), 179(7.2) \\
143(9.7), 161(21.6) \\
\operatorname{MS}^{4}[551 \rightarrow 505 \rightarrow 373]: 161(100)\end{array}$ & Unknown & - \\
\hline 52 & 10.6 & 515 & $\begin{array}{l}\operatorname{MS}^{2}[515]: 353(100), 335(15.8), 191(13), 179(24.4), 173(36) \\
\operatorname{MS}^{3}[515 \rightarrow 353]: 192(6.2), 191(48.8), 179(91.4), 173(100) \\
135(23.4) \\
\text { MS }^{4}[515 \rightarrow 353 \rightarrow 173]: 137(24.5), 129(16.1), 111(63), 94 \\
(16.6), 93(100)\end{array}$ & $\begin{array}{l}\text { 3,4-dicaffeoylquinic } \\
\text { acid }\end{array}$ & $\begin{array}{l}\text { Clifford et al. } \\
(2005)^{\mathrm{a}}\end{array}$ \\
\hline 56 & 11.6 & 515 & $\begin{array}{l}\operatorname{MS}^{2}[515]: 354(11.3), 353(100), 191(6.7) \\
\operatorname{MS}^{3}[515 \rightarrow 353]: 192(4.3), 191(100), 179(36.9), 135(8.3) \\
\operatorname{MS}^{4}[515 \rightarrow 353 \rightarrow 191]: 173(94.3), 127(38.2), 109(79.9), 93 \\
(100), 85(37.8)\end{array}$ & $\begin{array}{l}\text { 3,5-dicaffeoylquinic } \\
\text { acid }\end{array}$ & $\begin{array}{l}\text { Clifford et al. } \\
(2005)^{a}\end{array}$ \\
\hline 58 & 12.9 & 515 & $\begin{array}{l}\operatorname{MS}^{2}[515]: 354(15), 353(100), 203(13.7), 179(7.8), 173(29.6) \\
\operatorname{MS}^{3}[515 \rightarrow 353]: 191(21), 180(4.3), 179(57.5), 173(100), 135 \\
(15) \\
\text { MS }^{4}[515 \rightarrow 353 \rightarrow 173]: 155(35.4), 111(100), 93(76.7), 92 \\
(18.8), 69(15.9)\end{array}$ & $\begin{array}{l}\text { 4,5-dicaffeoylquinic } \\
\text { acid }\end{array}$ & $\begin{array}{l}\text { Clifford et al. } \\
(2005)^{a}\end{array}$ \\
\hline 69 & 17.4 & 515 & $\begin{array}{l}\mathrm{MS}^{2}[515]: 354(15), 353(100), 191(4.9), 179(8.3), 173(9.1) \\
\mathrm{MS}^{3}[515 \rightarrow 353]: 192(7.1), 191(63.2), 179(71.6), 173(100), \\
135(11.3) \\
\mathrm{MS}^{4}[515 \rightarrow 353 \rightarrow 173]: 155(58.2), 111(42), 109(22.8), 93 \\
(100), 83(31.5)\end{array}$ & Dicaffeoylquinic acid. & - \\
\hline 72 & 17.7 & 499 & $\begin{array}{l}\mathrm{MS}^{2}[499]: 338(16.9), 337(100), 173(24.9), 163(4.1) \\
\operatorname{MS}^{3}[499 \rightarrow 337]: 173(100), 163(15.4), 119(3.4) \\
\operatorname{MS}^{4}[499 \rightarrow 337 \rightarrow 173]: 137(20.9), 111(49.6), 109(21), 93 \\
(100), 71(14.9)\end{array}$ & $\begin{array}{l}\text { 3-O-caffeoyl-4-O-p- } \\
\text { coumaroylquinic } \\
\text { acid }\end{array}$ & $\begin{array}{l}\text { Clifford et al. } \\
(2006 a)\end{array}$ \\
\hline 75 & 20.1 & 493 & $\begin{array}{l}\operatorname{MS}^{2}[493]: 448(23.8), 447(100) \\
\operatorname{MS}^{3}[493 \rightarrow 447]: 315(100), 161(51.6), 159(13.2), 149(17.9) \\
131(19.7) \\
\operatorname{MS}^{4}[493 \rightarrow 447 \rightarrow 315]: 161(100), 159(7.6), 143(6), 113(4.5), \\
101(6.6)\end{array}$ & Unknown & - \\
\hline 76 & 21.5 & 583 & $\begin{array}{l}\text { MS }^{2}[583]: 538(10.7), 537 \text { (43.9), } 536(4.4), 430(18.6), 429 \\
(100) \\
\text { MS }^{3}[583 \rightarrow 429]: 369(6.4), 327 \text { (55.6), } 195(13.9), 153(100), \\
152(20.1) \\
\text { MS }^{4}[583 \rightarrow 429 \rightarrow 153]: 125(12.3), 110(16.1), 109(100), 108 \\
(56.7), 107(10.6)\end{array}$ & Unknown & - \\
\hline 77 & 21.8 & 839 & $\begin{array}{l}\operatorname{MS}^{2}[839]: 677(8.2), 665(3.6), 664(22.9), 663(100), 501(3.4) \\
\operatorname{MS}^{3}[839 \rightarrow 663]: 543(8.5), 502(19.1), 501(100), 484(7.2) \\
483(23.4), 439(4.8) \\
\text { MS }^{4}[839 \rightarrow 663 \rightarrow 501]: 484(14.4), 483(100), 441(5.1), 439 \\
(14.2), 437(6.1), 421(3.5)\end{array}$ & Triterpenoid saponin & - \\
\hline 84 & 27.8 & 795 & $\begin{array}{l}\operatorname{MS}^{2}[795]: 440(27.7), 439(100), 421(6.6) \\
\operatorname{MS}^{3}[795 \rightarrow 439]: 422(18.3), 421(100), 395(8.2) \\
\operatorname{MS}^{4}[795 \rightarrow 439 \rightarrow 421]: 376(22.1), 375(100)\end{array}$ & Unknown & - \\
\hline
\end{tabular}


Table 2 (Continued)

\begin{tabular}{|c|c|c|c|c|c|}
\hline No & $t_{R}(\min )$ & {$[\mathrm{M}-\mathrm{H}]^{-}(m / z)$} & HPLC-ESI-MS $; m / z$ (\% base peak) & Assigned identity & Ref. \\
\hline 91 & 31.8 & 327 & $\begin{array}{l}\mathrm{MS}^{2}[327]: 291 \text { (51.3), } 229(100), 211(74.8), 209(25.1), 171 \\
(72.8) \\
\text { MS }^{3}[327 \rightarrow 171]: 154(100), 127(63.5) \\
\operatorname{MS}^{3}[327 \rightarrow 229]: 211(100), 209(85.2), 165(51.3), 155(33.9) \\
125(25) \\
\operatorname{MS}^{4}[327 \rightarrow 229 \rightarrow 211]: 194(51.4), 175(100), 163(94.4)\end{array}$ & $\begin{array}{l}\text { Oxo-dihydroxy- } \\
\text { octadecenoic } \\
\text { acid }\end{array}$ & $\begin{array}{l}\text { Levandi et al. } \\
\text { (2009); Van } \\
\text { Hoyweghen } \\
\text { et al. (2014) }\end{array}$ \\
\hline 92 & 31.9 & 663 & $\begin{array}{l}\mathrm{MS}^{2}[663]: 543(24.6), 502(24.6), 501(100), 457(46.8), 439 \\
(22.2) \\
\mathrm{MS}^{3}[663 \rightarrow 501]: 484(17.4), 483(100), 457(25.5), 455(21.9), \\
439(13.7) \\
\mathrm{MS}^{4}[663 \rightarrow 501 \rightarrow 483]: 440(24.8), 439(100), 438(7.2), 437 \\
(31.3), 391(6.4)\end{array}$ & Triterpenoid saponin & $\begin{array}{l}\text { Chen et al. } \\
\text { (2011) }\end{array}$ \\
\hline 93 & 32.9 & 811 & $\begin{array}{l}\operatorname{MS}^{2}[811]: 765(4.7), 603(100), 604(33.6) \\
\operatorname{MS}^{3}[811 \rightarrow 603]: 586(34.3), 585(100) \\
\operatorname{MS}^{4}[811 \rightarrow 603 \rightarrow 585]: 571(36.8), 453(100)\end{array}$ & Unknown & - \\
\hline 95 & 33.9 & 811 & $\begin{array}{l}\operatorname{MS}^{2}[811]: 765(3.9), 603(100), 604(36.5) \\
\operatorname{MS}^{3}[811 \rightarrow 603]: 586(25.8), 585(100), 543(4.1) \\
\operatorname{MS}^{4}[811 \rightarrow 603 \rightarrow 585]: 571(100), 453(15.1)\end{array}$ & Unknown & - \\
\hline 97 & 34.5 & 895 & $\begin{array}{l}\operatorname{MS}^{2}[895]: 734(48.6), 733(100) \\
\operatorname{MS}^{3}[895 \rightarrow 733]: 571(100), 572(22.3), 553(6.5) \\
\operatorname{MS}^{4}[895 \rightarrow 733 \rightarrow 571]: 539(100), 439(17)\end{array}$ & Saponin-1 & $\begin{array}{l}\text { Khan et al. } \\
\text { (1993) }\end{array}$ \\
\hline 99 & 37.6 & 793 & 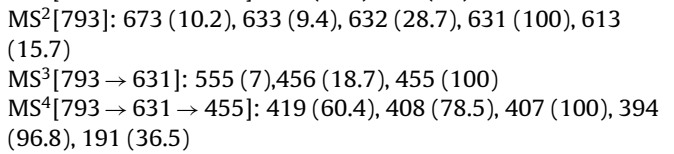 & Saponin-2 & $\begin{array}{l}\text { Gouveia and } \\
\text { Castilho (2011) }\end{array}$ \\
\hline 100 & 38 & 793 & $\begin{array}{l}\operatorname{MS}^{2}[793]: 633(7), 632(38.7), 631(100), 613(9.3), 569(4.6) \\
\operatorname{MS}^{3}[793 \rightarrow 631]: 555(8.2) 509(4.3), 457(6.7), 456(49.1), 455 \\
(100) \\
\operatorname{MS}^{4}[793 \rightarrow 631 \rightarrow 455]: 407(48.5), 191(100)\end{array}$ & Saponin-3 & $\begin{array}{l}\text { Gouveia and } \\
\text { Castilho (2011) }\end{array}$ \\
\hline 104 & 42.6 & 501 & 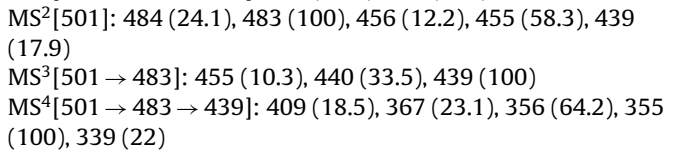 & Unknown & - \\
\hline 106 & 43.9 & 501 & $\begin{array}{l}\mathrm{MS}^{2}[501]: 484(18.7), 483(100), 456(11.1), 455(42.4), 439 \\
(17.9) \\
\mathrm{MS}^{3}[501 \rightarrow 483]: 455 \text { (34.3), } 440 \text { (35.6), } 439(100), 425(5.5) \\
423(8.8) \\
\mathrm{MS}^{4}[501 \rightarrow 483 \rightarrow 439]: 439 \text { (42.3), } 409(100), 367(66.4), 355 \\
(66.4), 309(46.8)\end{array}$ & Unknown & - \\
\hline
\end{tabular}

a Compared with standard compound.

the analysis of species of the Ilex genus, specifically I. paraguariensis (Bravo et al., 2007; Carini et al., 1998; Dartora et al., 2011). In these works, mono- and di-caffeoylquinic acids, along with other hydroxycinnamates, were the main detected compounds. In the present study, caffeoylquinic acids and other esters of quinic acid were also detected as the major components of Ilex perado (Table 2).

\subsubsection{Phenolic acids}

Compounds 6, 10, and 17, were identified as monocaffeoylquinic acids. All of them displayed $[\mathrm{M}-\mathrm{H}]^{-}$at $\mathrm{m} / \mathrm{z} 353$ and a dimer at $m / z$ 707. The MS ${ }^{n}$ fragmentation of the ion at $m / z$ 353 resulted in the typical fragmentation pattern of caffeoylquinic acids. The $\mathrm{MS}^{2}$ spectrum of compound $\mathbf{1 0}$ showed a fragment ion at $m / z 191$ as base peak and a relatively intense fragment ion at $m / z 179$. Based on the hierarchical key proposed by Clifford et al. (Clifford et al., 2003), compound $\mathbf{1 0}$ was characterized as 3-Ocaffeoylquinic acid. Compound $\mathbf{1 7}$ also showed base peak at $\mathrm{m} / \mathrm{z}$ 191 , but an undetectable fragment ion at $m / z 179$. Thus, it was identified as 5-O-caffeoylquinic acid. This identification was confirmed by the retention time when injecting the corresponding analytical standard. Compound $\mathbf{6}$ showed the same fragmentation pattern than 10, 3-caffeoylquinic acid, and could be an isomer, since UV exposure during sample preparation can sometimes induce some geometric isomerization in hydroxycinnamic acids (Clifford et al., 2006b). The presence of these caffeoylquinic acids was confirmed by the positive ionization mode, obtaining $[\mathrm{M}+\mathrm{H}]^{+}$at $\mathrm{m} / z 355$ with fragment ions at $m / z 163$ (base peak).

Compounds 52, 56, 58, and $\mathbf{6 9}$ exhibited $[\mathrm{M}-\mathrm{H}]^{-}$at $m / z 515$, and base peak in $\mathrm{MS}^{2}$ at $m / z 353$, [M-H-162]. They were all identified as di-caffeoylquinic acids. For their characterization, the hierarchical key for the identification of di-caffeoylquinic acids was used (Clifford et al., 2005), taking into account the different $\mathrm{MS}^{3}$ and $\mathrm{MS}^{4}$ spectra of the $m / z 353$ ions. Compounds 52, 58, and $\mathbf{6 9}$ presented $\mathrm{MS}^{3}$ base peak at $m / z 173$, which is indicative of a $4-\mathrm{OH}$ substituted quinic acid. Compound $\mathbf{5 2}$ showed weak (ca 15\%) MS ${ }^{2}$ ion at $m / z 335\left(15.8 \%\right.$ ) and strong ( $>50 \%$ base peak) $\mathrm{MS}^{3}$ ion $\mathrm{m} / \mathrm{z}$ 179 (91.4\%), being identified as 3-4-dicaffeoylquinic acid. Compound $\mathbf{5 8}$ presented undetectable $\mathrm{MS}^{2}$ ion at $\mathrm{m} / \mathrm{z} 335$ and strong ( $>50 \%$ base peak) ion at $\mathrm{m} / \mathrm{z} 179(57.5 \%$ ) and was characterized as 4-5-dicaffeoylquinic acid. Compound $\mathbf{5 6}$ was identified as 3,5dicaffeoylquinic acid, considering the $\mathrm{MS}^{3}$ base peak at $m / z 191$. These identifications were confirmed by the injection of analytical standards. Compound 69 showed a fragmentation pattern similar to 4-5-dicaffeoylquinic acid, but could not be fully characterized.

Compound 28 presented an $[\mathrm{M}-\mathrm{H}]^{-}$ion at $m / z 337$, with $\mathrm{MS}^{2}$ base peak at $m / z$ 173. According to the hierarchical key for identification of chlorogenic acids (Clifford et al., 2003), it was identified as 4-p-coumaroylquinic acid.

Compound 72 exhibited $[\mathrm{M}-\mathrm{H}]^{-}$ion at $m / z$ 499, and lost a caffeoyl moiety (162 Da) to form a base peak ion at $\mathrm{m} / \mathrm{z} 337$ in the $\mathrm{MS}^{2}$ spectrum. Considering the sequential MS ${ }^{\mathrm{n}}$ fragmentation 
<smiles>[Z]OC(=O)C1=COC(OC(O)C2OC(C)C(O)C(O)C2O)C(=CC)C1CC(=O)OCCc1ccc(O)c([R])c1</smiles>

Oleuropein

Demethyloleuropein Ligstroside
$\mathrm{R}_{1}=\mathrm{OH} ; \mathrm{R}_{2}=\mathrm{CH}_{3}$ $\mathrm{R}_{1}=\mathrm{OH} ; \mathrm{R}_{2}=\mathrm{H}$ $\mathrm{R}_{1}=\mathrm{H} ; \mathrm{R}_{2}=\mathrm{CH}_{3}$

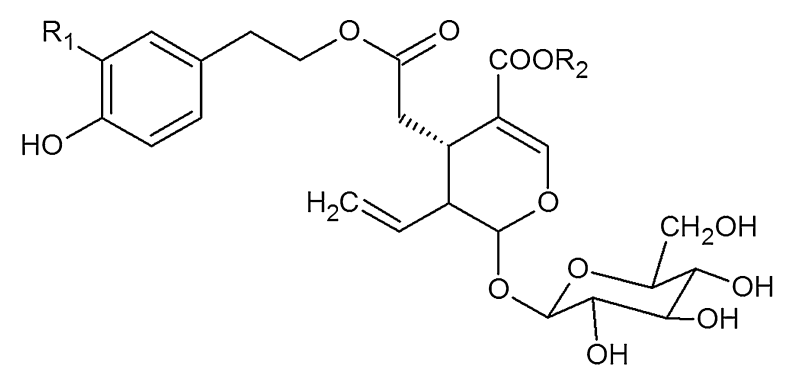

Oleuroside

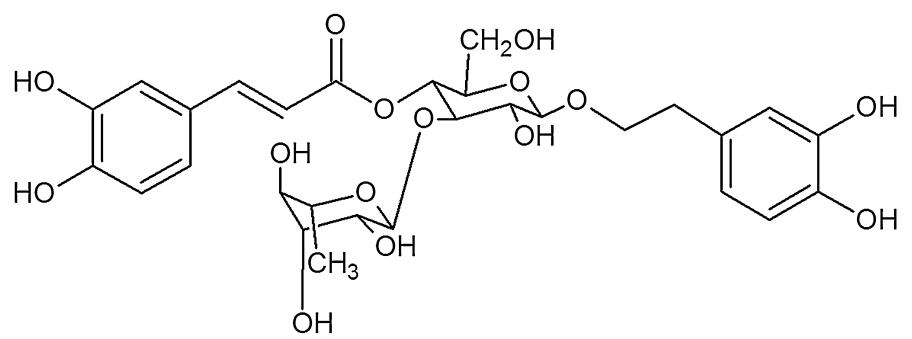<smiles>Oc1cc(O)c2c(c1)O[C@H](c1ccc(O)c(O)c1)C(O)C2</smiles><smiles>CC(C)(C)O[Mg]O[Mg]</smiles>

(-) Epicatechin<smiles>Oc1cc(O)c2c(c1)O[C@H](c1ccc(O)c(O)c1)[C@H](O)[C@H]2c1c(O)cc(O)c2c1O[C@H](c1ccc(O)c(O)c1)[C@H](O)C2</smiles><smiles>Oc1cc(O)c2c(c1)O[C@H](c1ccc(O)c(O)c1)[C@H](O)[C@H]2c1c(O)cc(O)c2c1O[C@H](c1ccc(O)c(O)c1)[C@H](O)[C@H]2c1c(O)cc(O)c2c1O[C@H](c1ccc(O)c(O)c1)[C@H](O)C2</smiles>

Procyanidin dimer

Procyanidin trimer

Fig. 4. Chemical structures of the main compounds found in the leaves of Olea europaea and Clethra arborea. 
pattern, the compound was identified as 3-0-caffeoyl-4-O-pcoumaroylquinic acid (Clifford et al., 2006a).

\subsubsection{Terpenoid saponins}

Compound 77, with $[\mathrm{M}-\mathrm{H}]^{-}$ion at $m / z$ 839, suffered the neutral loss of $176 \mathrm{Da}$ (probably glucuronide) in $\mathrm{MS}^{2}$ to yield a fragment ion at $m / z 663$ (base peak). In $\mathrm{MS}^{3}$, fragment ions at $m / z 501$ (base peak), 483, and 439 were observed. MS $^{4}$ fragmentation exhibited fragment ions at $m / z 483$ (base peak), 439, and 421. Compound 92, with $[\mathrm{M}-\mathrm{H}]^{-}$ion at $m / z 663$, displayed a similar fragmentation. This fragmentation pattern has been previously described for triterpenoids in Ilex species (Chen et al., 2011).

Compound 97 produced a $[\mathrm{M}-\mathrm{H}]^{-}$ion at $m / z$ 895. Fragment ions appeared at $m / z 733$ [M-H-162 $]^{-}$in $\mathrm{MS}^{2} ; 571$ [M-H-162-162 $]^{-}$in $\mathrm{MS}^{3}$; and 439 [M-H-162-162-132 $]^{-}$in $\mathrm{MS}^{4}$. This fragmentation pattern has been previously described for a saponin (Khan et al., 1993).

Compounds 99 and 100 presented $[\mathrm{M}-\mathrm{H}]^{-}$ions at $m / z$ 793, with ion fragments at $\mathrm{m} / z 631\left(\mathrm{MS}^{2}\right), 455\left(\mathrm{MS}^{3}\right)$, and 407 and 191 $\left(\mathrm{MS}^{4}\right)$. This fragmentation pattern has been previously reported for saponins (Gouveia and Castilho, 2011).

\subsubsection{Other compounds}

Compound 2, with $[\mathrm{M}-\mathrm{H}]^{-}$ion at $m / z 533$, showed a direct loss of $341 \mathrm{Da}$ at $\mathrm{MS}^{2}$, yielding a fragment ion at $m / z 191$, which presented a fragmentation pattern similar to quinic acid and, therefore, was characterized as a derivative.

Compound 75 displayed an $[\mathrm{M}-\mathrm{H}+\mathrm{HCOOH}]^{-}$ion at $m / z 493$. After loss of $46 \mathrm{Da}$ (formic) and $132 \mathrm{Da}$ (pentoside), it yielded a fragment ion at $m / z 315$, which probably corresponded to the aglycone. However, the exact nature of the aglycone could not be determined.

Compound 91, with a $[\mathrm{M}-\mathrm{H}]^{-}$ion at $m / z 327$ and $\mathrm{MS}^{2}$ base peak ion at $m / z 229$, was identified as oxo-dihydroxy octadecenoic acid (oxo-DHODE), an oxylipin (Levandi et al., 2009; Van Hoyweghen et al., 2014).

Compounds 93 and 95 showed similar mass spectra presenting $[\mathrm{M}-\mathrm{H}+\mathrm{HCOOH}]^{-}$at $m / z$ 811. In $\mathrm{MS}^{2}$, they suffered a neutral loss of $208 \mathrm{Da}(46+162)$, which could correspond to the loss of formic acid and a hexoside residue, yielding a fragment ion at $m / z$ 603. In $\mathrm{MS}^{3}$, they suffered a loss of $18 \mathrm{Da}$ (water) and yielded an ion at $m / z 585$, which suffered further loss producing ions at $m / z 571$ ( $-14 \mathrm{Da}$ ) and $453(-132 \mathrm{Da})$. These two compounds may be isomers, but their nature could not be elucidated due to the absence of bibliographic data.

\subsection{Heberdenia excelsa}

To our best knowledge, there are not bibliographic data to compare the obtained results in the analysis of Heberdenia leaves. Most of the detected compounds corresponded to phenolic acids, flavonoids (mainly rhamnosides) and isoflavonoids, although other compounds such as phenylethanoid glycosides were identified (Table 3).

\subsubsection{Phenolic acids}

Compound 11 displayed $[\mathrm{M}-\mathrm{H}]^{-}$at $m / z 385$, and in $\mathrm{MS}^{2}$ suffered the loss of the glucose unit and the loss of the acid group from the aglycone, yielding the fragment ions at $m / z 223$ and 179 . It was identified as sinapic acid-O-glucoside (Sánchez-Rabaneda et al., 2004). Compound 32 also exhibited fragment ions at $\mathrm{m} / \mathrm{z} 223$ and 179 in its $\mathrm{MS}^{\mathrm{n}}$ fragmentation pattern, and was characterized as a sinapic acid derivative.

Compound 27, with $[\mathrm{M}-\mathrm{H}]^{-}$at $m / z 435$, showed in his fragmentation characteristic ions of protocatechuic acid, at $m / z 153$ and 109 (Gouveia and Castilho, 2012), and was tentatively identified as a derivative.
Compound 48 showed $[\mathrm{M}-\mathrm{H}]^{-}$at $m / z$ 247. It suffered a neutral loss of $80 \mathrm{Da}$ (sulphate) in $\mathrm{MS}^{2}$, yielding a fragment ion at $m / z 167$, which displayed a fragmentation pattern typical from vanillic acid. It was therefore characterized as vanillic acid sulphate, taking into account bibliographic data (Suárez et al., 2011).

\subsubsection{Flavonoids}

Glycosylated flavonoids from myricetin, kaempferol, mearnsetin, and quercetin were identified. Compounds $\mathbf{4 2}$, $\mathbf{5 5}$, and $\mathbf{6 5}$ displayed deprotonated molecular ions at $\mathrm{m} / \mathrm{z} 463,447$, and 431 , respectively. All of them suffered the neutral loss of a rhamnoside unit ( $146 \mathrm{Da}$ ) in $\mathrm{MS}^{2}$, yielding fragment ions that corresponding to the aglycones myricetin, quercetin, and kaempferol. Compound 54, with $[\mathrm{M}-\mathrm{H}]^{-}$ion at $m / z$ 477, also suffered the neutral loss of $146 \mathrm{Da}$ in $\mathrm{MS}^{2}$ producing a fragment ion at $\mathrm{m} / z 331$, which corresponded to mearnsetin (typical fragment ion at $m / z$ 316) (Han et al., 2008). Hence, the compound was characterized as mearnsetin-O-rhamnoside.

\subsubsection{Isoflavonoids}

Compound 15, with $[\mathrm{M}-\mathrm{H}]^{-}$at $m / z 429$, lost $162 \mathrm{Da}$ (hexoside) in $\mathrm{MS}^{2}$, yielding a fragment ion at $m / z 267$. Compound 22 showed $[\mathrm{M}-\mathrm{H}]^{-}$ion at $m / z 581$ and suffered the neutral loss of $152 \mathrm{Da}$ (galloyl moiety) and $162 \mathrm{Da}$ (hexoside), producing also the aglycone at $\mathrm{m} / \mathrm{z} 267$. Compound $\mathbf{3 6}$ also produced the same aglycone after several fragmentations. According to Prasain et al. (2003), the aglycone is most likely formononetin, a methoxylated isoflavonoid. Compound 15 showed a similar pattern to formononetin-7-O-glucoside and was identified as such. Compounds $\mathbf{2 2}$ and $\mathbf{3 6}$ were characterized as formononetin derivatives.

\subsubsection{Other compounds}

Compounds $\mathbf{3 8}$ and $\mathbf{4 5}$ exhibited the same deprotonated molecular ion, at $m / z 623$, and same $\mathrm{MS}^{\mathrm{n}}$ fragmentation, yielding ions at $m / z 461,315$, and 135 . This fragmentation pattern has been previously described for phenylethanoid glycosides (Guo et al., 2007). Considering their retention time, $\mathbf{3 8}$ and $\mathbf{4 5}$ were identified as acteoside and forsythoside $\mathrm{A}$, respectively

Compound 60 displayed the deprotonated molecular ion at $m / z 287$, and suffered the loss of $80 \mathrm{Da}$ (sulphate) in $\mathrm{MS}^{2}$. This fragmentation has been previously reported for dihydroxyphenylvalerolactone sulphates (Urpi-Sarda et al., 2009a,b).

Compounds 66 and 70 showed $[\mathrm{M}-\mathrm{H}]^{-}$ion at $m / z$ 457, and displayed the main fragment ions at $m / z 260,231$, and 97 . Considering their similar fragmentation patterns, they can be considered isomers. This fragmentation has been previously reported in leaves from Helichrysum melaleucum (Gouveia and Castilho, 2010), but their identification could not be carried out.

Compound 96 was identified as trihydroxy-octadecenoic acid, considering its $[\mathrm{M}-\mathrm{H}]^{-}$ions at $\mathrm{m} / \mathrm{z} 329$ and its fragmentation pattern (Van Hoyweghen et al., 2014).

\subsection{Clethra arborea}

The main groups of compounds found during the analysis of the extracts from Clethra leaves were phenolic acids, procyanidins (dimers and trimers), and flavonoids, mainly glucuronides. The results $\left(\mathrm{ESI}^{-}\right)$are shown in Table 4 . ESI ${ }^{+}$was also used for confirmation purposes and for the identification of anthocyanidins.

\subsubsection{Phenolic acids}

Compound 25, with $[\mathrm{M}-\mathrm{H}]^{-}$at $m / z$ 311, displayed both tartaric and caffeic acid as $\mathrm{MS}^{2}$ fragment ions at $m / z 149$ and 179, respectively. Fragment ions at $m / z 135$ (decarboxylated caffeic acid) and $m / z 87$ (characteristic of tartaric acid) were observed in $\mathrm{MS}^{3}$. 
Table 3

Characterization of the methanolic extracts from leaves of Heberdenia excelsa.

\begin{tabular}{|c|c|c|c|c|c|}
\hline No & $t_{R}(\min )$ & {$[\mathrm{M}-\mathrm{H}]^{-}(m / z)$} & HPLC-ESI-MS $; m / z$ (\% base peak) & Assigned identity & Ref. \\
\hline 1 & 2.7 & 683 & $\begin{array}{l}\mathrm{MS}^{2}[683]: 341(100) \\
\mathrm{MS}^{3}[683 \rightarrow 341]: 179(100), 161(19.8), 143(90.9), 131(29.9) \\
119(28.0), 113(26.4) \\
\mathrm{MS}^{4}[683 \rightarrow 341 \rightarrow 179]: 161(38.1), 101(100), 89(71.4)\end{array}$ & Hexose polymer & $\begin{array}{l}\text { Brudzynski and } \\
\text { Miotto (2011) }\end{array}$ \\
\hline 2 & 2.8 & 533 & $\begin{array}{l}\operatorname{MS}^{2}[533]: 191(100) \\
\operatorname{MS}^{3}[533 \rightarrow 191]: 173 \text { (49.6), } 127(50.1), 111(32.4), 93(100) \\
85(91.6)\end{array}$ & Quinic acid derivative & - \\
\hline 3 & 2.8 & 549 & $\begin{array}{l}\operatorname{MS}^{2}[549]: 503(100) \\
\operatorname{MS}^{3}[549 \rightarrow 503]: 323(18.2), 221(60.7), 179(100), 143(11.8) \\
\text { MS }^{4}[549 \rightarrow 503 \rightarrow 179]: 161(100), 143(20.9), 119(92), 101 \\
(59.5)\end{array}$ & Hexose polymer & $\begin{array}{l}\text { Brudzynski and } \\
\text { Miotto (2011) }\end{array}$ \\
\hline 4 & 3 & 191 & $\begin{array}{l}\operatorname{MS}^{2}[191]: 173(86.7), 127(79.5), 111(100), 93(38.2), 85(57.5) \\
\operatorname{MS}^{3}[191 \rightarrow 111]: 93(100) \\
\operatorname{MS}^{3}[191 \rightarrow 127]: 109(100)\end{array}$ & Quinic acid & $\begin{array}{l}\text { Gouveia and } \\
\text { Castilho (2011) }\end{array}$ \\
\hline 8 & 3.9 & 317 & $\begin{array}{l}\operatorname{MS}^{2}[317]: 225(100), 165(25.6), 153(14.7), 125(18) \\
\operatorname{MS}^{3}[317 \rightarrow 225]: 207(93.1), 165(100), 143(40.5), 125(59.1) \\
81(20)\end{array}$ & Unknown & - \\
\hline 11 & 4.2 & 385 & $\begin{array}{l}\operatorname{MS}^{2}[385]: 223(100), 179(70.4) \\
\operatorname{MS}^{3}[385 \rightarrow 223]: 179(100) \\
\operatorname{MS}^{4}[385 \rightarrow 223 \rightarrow 179]: 150(100), 138(79.4), 124(91.3), 123 \\
(61.9), 109(92.6)\end{array}$ & Sinapic acid-O-glucoside & $\begin{array}{l}\text { (Sánchez- } \\
\text { Rabaneda et al. } \\
\text { (2004) }\end{array}$ \\
\hline 15 & 4.4 & 429 & $\begin{array}{l}\operatorname{MS}^{2}[429]: 267(100), 249(25.7), 231(12.3) \\
\text { MS }^{3}[429 \rightarrow 267]: 249(100), 231(35), 205(11.8), 139(10.4) \\
\text { MS }^{4}[429 \rightarrow 267 \rightarrow 249]: 231(100), 205(19), 187(24.7)\end{array}$ & Formononetin-7-O-glucoside & $\begin{array}{l}\text { Prasain et al. } \\
(2003)\end{array}$ \\
\hline 22 & 5.5 & 581 & $\begin{array}{l}\mathrm{MS}^{2}[581]: 430(14.9), 429(100), 313(29.1), 267(80.6), 249 \\
(11.6) \\
\text { MS }^{3}[581 \rightarrow 429]: 267(100), 249(16.3), 231(14.3) \\
\text { MS }^{4}[581 \rightarrow 429 \rightarrow 267]: 249(100), 231(34.7)\end{array}$ & Formononetin derivative & - \\
\hline 27 & 6.2 & 435 & $\begin{array}{l}\operatorname{MS}^{2}[435]: 298(15.1), 297(100), 153(44.9) \\
\text { MS }^{3}[435 \rightarrow 297]: 195(12.3), 177(12.7), 153(100), 109(12.9) \\
\text { MS }^{4}[435 \rightarrow 297 \rightarrow 153]: 109(100), 108(15.1)\end{array}$ & Protocatechuic acid derivative & - \\
\hline 32 & 7.1 & 521 & $\begin{array}{l}\operatorname{MS}^{2}[521]: 477(16.4), 255(16.4), 223(100), 205(29), 179 \\
(61.7) \\
\text { MS }^{3}[521 \rightarrow 223]: 179(100), 163(9.3), 161(9.2) \\
\text { MS }^{3}[521 \rightarrow 179]: 138(77.2), 137(27.9), 125(33.1), 124(100) \\
\operatorname{MS}^{4}[521 \rightarrow 223 \rightarrow 179]: 151(86.9), 138(99.7), 124(29.8)\end{array}$ & Sinapic acid derivative & - \\
\hline 36 & 7.3 & 565 & $\begin{array}{l}\operatorname{MS}^{2}[565]: 429(22.7), 297(15.6), 267(100), 249(35.2), 231 \\
(22.6) \\
\operatorname{MS}^{3}[565 \rightarrow 267]: 249(100), 231(40.8), 205(16.5), 187(14.2) \\
\operatorname{MS}^{4}[565 \rightarrow 267 \rightarrow 249]: 231(100)\end{array}$ & Formononetin derivative & - \\
\hline 38 & 7.6 & 623 & $\begin{array}{l}\operatorname{MS}^{2}[623]: 477(1.4), 461(100) \\
\operatorname{MS}^{3}[623 \rightarrow 461]: 315(93.9), 297(17), 161(16.7), 151(15.1) \\
135(100) \\
\operatorname{MS}^{4}[623 \rightarrow 461 \rightarrow 315]: 135(100), 91(76.6)\end{array}$ & Acteoside & $\begin{array}{l}\text { Guo et al. } \\
(2007)\end{array}$ \\
\hline 42 & 8.2 & 463 & $\begin{array}{l}\operatorname{MS}^{2}[463]: 317(77.3), 316(100) \\
\operatorname{MS}^{3}[463 \rightarrow 316]: 287(15), 271(100), 270(35), 179(67.6), 151 \\
(14.9) \\
\text { MS }^{4}[463 \rightarrow 316 \rightarrow 179]: 151(66.2) \\
\operatorname{MS}^{4}[463 \rightarrow 316 \rightarrow 271]: 244(27.4), 243(52.5), 242(100), 215 \\
(24.5)\end{array}$ & Myricetin-O-rhamnoside & - \\
\hline 45 & 9 & 623 & $\begin{array}{l}\operatorname{MS}^{2}[623]: 462(22.1), 461(100) \\
\text { MS }^{3}[623 \rightarrow 461]: 315(44.8), 297(12.2), 135(100) \\
\text { MS }^{4}[623 \rightarrow 461 \rightarrow 315]: 135(100)\end{array}$ & Forsythoside A & $\begin{array}{l}\text { Guo et al. } \\
\text { (2007) }\end{array}$ \\
\hline 48 & 9.6 & 247 & $\begin{array}{l}\operatorname{MS}^{2}[247]: 167(100), 139(22.4), 123(51.2) \\
\operatorname{MS}^{3}[247 \rightarrow 167]: 123(100) \\
\operatorname{MS}^{4}[247 \rightarrow 167 \rightarrow 123]: 81(100)\end{array}$ & Vanillic acid sulphate & $\begin{array}{l}\text { Suárez et al. } \\
\text { (2011) }\end{array}$ \\
\hline 54 & 11.2 & 477 & $\begin{array}{l}\operatorname{MS}^{2}[477]: 331(100), 316(26.5), 315(24.3) \\
\operatorname{MS}^{3}[477 \rightarrow 331]: 316(100) \\
\operatorname{MS}^{4}[477 \rightarrow 331 \rightarrow 316]: 287(45.1), 271(95.7), 179(100), 164 \\
(24.6)\end{array}$ & Mearnsetin-O-rhamnoside & - \\
\hline 55 & 11.3 & 447 & $\begin{array}{l}\text { MS }^{2}[447]: 302(14.2), 301(100), 300(31.4) \\
\text { MS }^{3}[447 \rightarrow 301]: 271(24.7), 255(17.5), 179(100), 151(85.5) \\
\text { MS }^{4}[447 \rightarrow 301 \rightarrow 179]: 169(16.3), 151(100)\end{array}$ & Quercetin-O-rhamnoside & - \\
\hline 60 & 13.5 & 287 & $\begin{array}{l}\operatorname{MS}^{2}[287]: 208(14.3), 207(100) \\
\operatorname{MS}^{3}[287 \rightarrow 207]: 163 \text { (92.1), } 123 \text { (100), } 121 \text { (10.5) }\end{array}$ & $\begin{array}{l}\text { Dihydroxyphenyl- } \\
\text { valerolactone } \\
\text { sulphate }\end{array}$ & $\begin{array}{l}\text { Urpi-Sarda } \\
\text { et al. (2009a,b) }\end{array}$ \\
\hline 65 & 15.1 & 431 & $\begin{array}{l}\mathrm{MS}^{2}[431]: 286 \text { (16.9), } 285 \text { (100), } 284(24.6) \\
\mathrm{MS}^{3}[431 \rightarrow 285]: 257(50.6), 256(35.1), 255(100), 229(22) \\
213(14.3) \\
\mathrm{MS}^{4}[431 \rightarrow 285 \rightarrow 256]: 239(36.5), 229(92.7), 227(100), 213 \\
(40.2), 163(70.4)\end{array}$ & Kaempferol-O-rhamnoside & - \\
\hline 66 & 15.7 & 457 & $\begin{array}{l}\mathrm{MS}^{2}[457]: 329(28.3), 261(17.9), 260(100) \\
\operatorname{MS}^{3}[457 \rightarrow 260]: 245 \text { (21.5), } 231 \text { (34.9), } 179(16.8), 110(17.9) \\
97(100)\end{array}$ & Unknown & $\begin{array}{l}\text { Gouveia and } \\
\text { Castilho (2010) }\end{array}$ \\
\hline
\end{tabular}


Table 3 (Continued)

\begin{tabular}{|c|c|c|c|c|c|}
\hline No & $t_{R}(\min )$ & {$[\mathrm{M}-\mathrm{H}]^{-}(m / z)$} & HPLC-ESI-MS $; m / z$ (\% base peak) & Assigned identity & Ref. \\
\hline 67 & 15.9 & 499 & 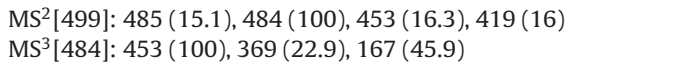 & Unknown & - \\
\hline 70 & 17.5 & 457 & $\begin{array}{l}\mathrm{MS}^{2}[457]: 275(92.5), 261(20.1), 260(100), 231(10.1) \\
\mathrm{MS}^{3}[457 \rightarrow 260]: 245(10.7), 231(63.4), 179(35), 110(27.6), \\
97(100)\end{array}$ & Unknown & $\begin{array}{l}\text { Gouveia and } \\
\text { Castilho (2010) }\end{array}$ \\
\hline 71 & 17.5 & 439 & $\begin{array}{l}\mathrm{MS}^{2}[439]: 425 \text { (25.4), } 424(100), 393(11.6), 359(52.3), 316 \\
(12.3) \\
\text { MS }^{3}[439 \rightarrow 424]: 394 \text { (44.2), } 393(100), 312(44.2)\end{array}$ & Unknown & - \\
\hline 74 & 20.1 & 523 & $\begin{array}{l}\mathrm{MS}^{2}[523]: 362(24.9), 361(100), 292(14.2), 291(92), 259 \\
(52.4) \\
\operatorname{MS}^{3}[523 \rightarrow 361]: 292(18.6), 291(100), 259(77.5) \\
\text { MS }^{4}[523 \rightarrow 361 \rightarrow 291]: 259(22.9), 231(19.8), 171(11.1), 139 \\
(58.5), 127(14.6), 111(100)\end{array}$ & Ligstroside & $\begin{array}{l}\text { Briante et al. } \\
\text { (2002); } \\
\text { Laguerre et al. } \\
\text { (2009) }\end{array}$ \\
\hline 85 & 28.8 & 305 & $\begin{array}{l}\mathrm{MS}^{2}[305]: 290(100), 275(18.3), 111(14.4), 97(41.8) \\
\mathrm{MS}^{3}[305 \rightarrow 290]: 275(100), 209(48.8), 195(14.1), 97(44.4) \\
\text { MS }^{4}[305 \rightarrow 290 \rightarrow 275]: 195(100), 97(80.1)\end{array}$ & Unknown & - \\
\hline 87 & 30.4 & 601 & $\begin{array}{l}\operatorname{MS}^{2}[601]: 556(23), 555(100), 393(42.4) \\
\operatorname{MS}^{3}[601 \rightarrow 555]: 394(16.3), 393(100) \\
\operatorname{MS}^{4}[601 \rightarrow 555 \rightarrow 393]: 209(11.1), 183(100), 139(16.1)\end{array}$ & Unknown & - \\
\hline 89 & 31.8 & 439 & $\begin{array}{l}\operatorname{MS}^{2}[439]: 421(20.2), 410(19), 409(100) \\
\text { MS }^{3}[439 \rightarrow 409]: 394(53.3), 330(12.8), 329(100), 314(38.9) \\
\operatorname{MS}^{4}[439 \rightarrow 409 \rightarrow 329]: 315(11.6), 314(100)\end{array}$ & Unknown & - \\
\hline 94 & 33.2 & 439 & $\begin{array}{l}\mathrm{MS}^{2}[439]: 409(100), 299(26.9), 259(22.0), 223(16.0) \\
\text { MS }^{3}[439 \rightarrow 409]: 395 \text { (83.2), } 329(100), 315(94.3), 314(64.6)\end{array}$ & Unknown & - \\
\hline 96 & 34.3 & 329 & 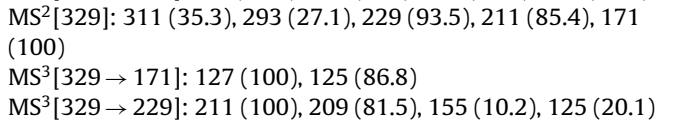 & Trihydroxy-octadecenoic acid & $\begin{array}{l}\text { Van } \\
\text { Hoyweghen } \\
\text { et al. (2014) }\end{array}$ \\
\hline
\end{tabular}

This fragmentation was consistent with caftaric acid (Schütz et al., 2005).

For the characterization of compound 53, the positive-ion mode was used due to the more informative fragment ions. This compound presented the protonated molecular ion at $\mathrm{m} / \mathrm{z} 373$, and fragment ions at $m / z 211,193,175$ and 135 . The fragment ions at $\mathrm{m} / \mathrm{z} 211$ and 193 corresponded to the losses of a dehydrated hexose and a hexose, respectively. The ions at $\mathrm{m} / \mathrm{z} 193,175$ and 135 were indicative of a hydroxyferulic acid residue. Hence, this compound was tentatively characterized as a hydroxyferulic acid glycoside (Ma et al., 2007).

Compound 62 was characterized as rosmarinic acid, considering its $[\mathrm{M}-\mathrm{H}]^{-}$ion at $m / z 359$, and $\mathrm{MS}^{\mathrm{n}}$ fragment ions at $m / z 197,179$, 161, and 133 (Liu et al., 2007).

\subsubsection{Procyanidins}

Several procyanidin dimers and trimers were detected in the analyzed extracts. Procyanidin dimers $(\mathbf{7}, \mathbf{1 8}, \mathbf{3 9})$ showed characteristic $[\mathrm{M}-\mathrm{H}]^{-}$ions at $m / z 577$, with $\mathrm{MS}^{2}$ fragment ions at $m / z$ $451,425,407,289$, and 287. Procyanidin trimers $(\mathbf{1 6}, \mathbf{2 1}, \mathbf{3 5})$ displayed $[\mathrm{M}-\mathrm{H}]^{-}$ions at $m / z$ 865, and typical $\mathrm{MS}^{2}$ fragment ions at $m / z 739,713,695,577,407$, and 289 . These fragment patterns have been previously reported (Ruiz et al., 2005; Tomás-Barberán et al., 2001). In addition, $[\mathrm{M}+\mathrm{H}]^{+}$ions at $m / z 579$ and 865 were observed in $\mathrm{ESI}^{+}$for procyanidin dimers and trimers, respectively, confirming the results obtained in $\mathrm{ESI}^{-}$.

\subsubsection{Flavonoids}

Compound 23 was characterized as catechin, based on its $[\mathrm{M}-\mathrm{H}]^{-}$ion at $m / z 289$ and the comparison of its fragmentation pattern with bibliographic data (Rockenbach et al., 2012). Compound 78, with $[\mathrm{M}-\mathrm{H}]^{-}$at $m / z 543$, suffered a neutral loss of $288 \mathrm{Da}$, yielding a fragment ion at $m / z 255$. This loss is probably due to an (epi)catechin unit (González-Paramás et al., 2006; Mateos-Martín et al., 2012). However, the exact nature of the compound could not be elucidated.
Rutin (compound 33) was identified, using an analytical standard, due to its characteristic $[\mathrm{M}-\mathrm{H}]^{-}$ion at $m / z 609$ and $\mathrm{MS}^{2}$ base peak at $m / z 301$.

Compound 41 exhibited $[\mathrm{M}-\mathrm{H}]^{-}$ion at $m / z 607$ and suffered the neutral loss of $322 \mathrm{Da}$, yielding a fragment ion at $m / z 285$, which corresponded to kaempferol (Ye et al., 2005). The exact nature of this compound could not be determined and it was identified as a kaempferol derivative. Compound $\mathbf{4 3}$ displayed an $[\mathrm{M}-\mathrm{H}]^{-}$ion at $m / z 593$ and yielded an MS $^{2}$ fragment ion at $m / z 285$ by loss of $308 \mathrm{Da}$ (hexose-rhamnose), and was identified as kaempferol 3-rutinoside (Del Rio et al., 2004). The fragmentation pattern of kaempferol was also confirmed by the analysis of a commercial standard.

Several glucuronides were identified in Clethra extracts. Compound 49 exhibited an $[\mathrm{M}-\mathrm{H}]^{-}$ion at $m / z 477$ and suffered a neutral loss of $176 \mathrm{Da}$ (glucuronide), yielding the aglycone at $\mathrm{m} / \mathrm{z} 301$. The fragmentation of the ion at $m / z 301$ yielded fragments at $m / z 273$, 257,179 , and 151, typical from quercetin, and therefore it was identified as a quercetin glucuronide (Downey and Rochfort, 2008). Compound $\mathbf{5 0}$ also suffered a neutral loss of $176 \mathrm{Da}$ in $\mathrm{MS}^{2}$, yielding a fragment ion at $m / z 287$ (eriodictyol), so it was identified as eriodictyol glucuronide (Fabre et al., 2001). Compound 63, with the aglycone at $m / z 271$, was characterized as naringenin glucuronide (Sánchez-Rabaneda et al., 2004).

\subsubsection{Other compounds}

Oleuropein (compound 61) was identified, as previously discussed, based on its $[\mathrm{M}-\mathrm{H}]^{-}$at $m / z 539$, and fragment ions at $m / z$ 377, 307, and 275.

Compounds 91 and 96 were characterized as oxo-dihydroxyoctadecenoic and trihydroxy-octadecenoic acids, respectively, taking into account the fragmentation data, previously described in scientific literature (Levandi et al., 2009; Van Hoyweghen et al., 2014).

Using $\mathrm{ESI}^{+}$, it was possible to characterize compound $\mathbf{3 4}$ as delphinidin glucuronide, showing $\mathrm{M}^{+}$ion at $\mathrm{m} / z 479$ and suffering a neutral loss of $176 \mathrm{Da}$ in $\mathrm{MS}^{2}$. 
Table 4

Characterization of the methanolic extracts from leaves of Clethra arborea.

\begin{tabular}{|c|c|c|c|c|c|}
\hline No & $t_{R}(\min )$ & {$[\mathrm{M}-\mathrm{H}]^{-}(m / z)$} & HPLC-DAD-MS $; m / z$ (\% base peak) & Assigned identity & Ref. \\
\hline 1 & 2.7 & 683 & $\begin{array}{l}\mathrm{MS}^{2}[683]: 341(100) \\
\mathrm{MS}^{3}[683 \rightarrow 341]: 179(100), 161(11.4), 143(20.3), 119(36.3) \\
113(33.9) \\
\mathrm{MS}^{4}[683 \rightarrow 341 \rightarrow 179]: 161(18.2), 119(15.1), 115(15.5), 107 \\
(26.6), 89(100)\end{array}$ & Hexose polymer & $\begin{array}{l}\text { Brudzynski and } \\
\text { Miotto (2011) }\end{array}$ \\
\hline 2 & 2.8 & 533 & $\begin{array}{l}\operatorname{MS}^{2}[533]: 191(100) \\
\operatorname{MS}^{3}[533 \rightarrow 191]: 173(46.7), 171(26), 127(100), 109(39.9), 85 \\
(42.7) \\
\operatorname{MS}^{4}[533 \rightarrow 191 \rightarrow 127]: 110(100), 109(17.3), 85(71.8)\end{array}$ & Quinic acid derivative & - \\
\hline 4 & 3.2 & 191 & 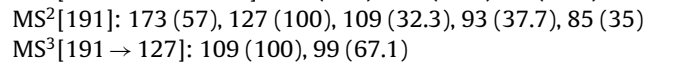 & Quinic acid & $\begin{array}{l}\text { Gouveia and } \\
\text { Castilho (2011) }\end{array}$ \\
\hline 7 & 3.2 & 577 & $\begin{array}{l}\mathrm{MS}^{2}[577]: 451(19.5), 425(100), 407(92.4), 289(23.6), 287 \\
(7.5) \\
\text { MS }^{3}[577 \rightarrow 407]: 389(31.3), 285(100), 284(25.2), 283(46.1), \\
281(53.1) \\
\text { MS }^{4}[577 \rightarrow 407 \rightarrow 284]: 283(23.1), 257(100), 255(14.6), 241 \\
(23.1), 213(35.5) \\
\text { MS }^{3}[577 \rightarrow 425]: 408(15.2), 407(100) \\
\text { MS}^{4}[577 \rightarrow 425 \rightarrow 407]: 285(77.9), 281(100), 256(28.3), 255 \\
(25), 243(39.2)\end{array}$ & Procyanidin dimer & $\begin{array}{l}\text { Ruiz et al. (2005); } \\
\text { Tomás-Barberán } \\
\text { et al. (2001) }\end{array}$ \\
\hline 16 & 4.5 & 865 & $\begin{array}{l}\mathrm{MS}^{2}[865]: 739(26.9), 713(27.9), 695(100), 577(64.9), 575 \\
(45.8), 407(58), 289(10.4) \\
\mathrm{MS}^{3}[865 \rightarrow 695]: 677(40.2), 544(32.5), 543(100), 525(70.3) \\
243(74.8) \\
\text { MS }^{4}[865 \rightarrow 695 \rightarrow 543]: 526(37.7), 525(100), 499(15), 404 \\
(13.9), 392(18.3) \\
\text { MS }^{4}[865 \rightarrow 695 \rightarrow 677]: 610(11.5), 600(100), 555(12), 554 \\
(48.6), 467(7)\end{array}$ & Procyanidin trimer & $\begin{array}{l}\text { Ruiz et al. (2005); } \\
\text { Tomás-Barberán } \\
\text { et al. (2001) }\end{array}$ \\
\hline 18 & 4.7 & 577 & $\begin{array}{l}\mathrm{MS}^{2}[577]: 451(20.1), 425(100), 407(95.5), 289(26.2), 287 \\
(14.2) \\
\mathrm{MS}^{3}[577 \rightarrow 407]: 285(100), 281(65.3), 257(46.3), 255(37.4) \\
243(35.6) \\
\mathrm{MS}^{4}[577 \rightarrow 407 \rightarrow 285]: 283(100), 257(51.6), 256(54.1),(155 \\
(10.8) \\
\mathrm{MS}^{3}[577 \rightarrow 425]: 408(18.8), 407(100) \\
\mathrm{MS}^{4}[577 \rightarrow 425 \rightarrow 407]: 285(50.8), 283(29.9), 281(100), 257 \\
(29.3), 256(33.8)\end{array}$ & Procyanidin dimer & $\begin{array}{l}\text { Ruiz et al. (2005); } \\
\text { Tomás-Barberán } \\
\text { et al. (2001) }\end{array}$ \\
\hline 21 & 5.4 & 865 & $\begin{array}{l}\mathrm{MS}^{2}[865]: 739(28.6), 713(27.1), 695(100), 577(85), 425 \\
(30.9), 407(53.6), 289(7.8) \\
\text { MS }^{3}[865 \rightarrow 695]: 543(100), 525(64.8), 451(30.6), 405(29.8), \\
243(54.8) \\
\text { MS }^{4}[865 \rightarrow 695 \rightarrow 543]: 526(31.5), 525(100), 405 \text { (8.6), } 391 \\
(26), 243(9)\end{array}$ & Procyanidin trimer & $\begin{array}{l}\text { Ruiz et al. (2005); } \\
\text { Tomás-Barberán } \\
\text { et al. (2001) }\end{array}$ \\
\hline 23 & 5.6 & 289 & $\begin{array}{l}\mathrm{MS}^{2}[289]: 247(14.5), 246(10.9), 245 \text { (100), } 205 \text { (36.5), } 179 \\
(16.2) \\
\mathrm{MS}^{3}[289 \rightarrow 245]: 227(11.8), 203(100), 187(16.8), 175(7.7), \\
161(13.6) \\
\mathrm{MS}^{4}[289 \rightarrow 245 \rightarrow 203]: 188(35.3), 175(16.3), 174(100), 161 \\
(53.3), 160(15.9)\end{array}$ & Catechin & $\begin{array}{l}\text { Rockenbach et al. } \\
\text { (2012) }\end{array}$ \\
\hline 25 & 5.8 & 311 & $\begin{array}{l}\mathrm{MS}^{2}[311]: 179(44.5), 177(34.1), 149(100), 135(5.9) \\
\mathrm{MS}^{3}[311 \rightarrow 149]: 131 \text { (71.6), } 103 \text { (66.5), } 87(100), 59(18.4)\end{array}$ & Caftaric acid & Schütz et al. (2005) \\
\hline 33 & 6.9 & 609 & $\begin{array}{l}\operatorname{MS}^{2}[609]: 307(12.6), 301(100) \\
\operatorname{MS}^{3}[609 \rightarrow 301]: 273(9.6), 229(10.5), 179(100), 151(94.3), \\
107(11.1) \\
\operatorname{MS}^{4}[609 \rightarrow 301 \rightarrow 179]: 169(19.7), 151(100)\end{array}$ & Rutin & - \\
\hline 34 & 7.0 & $479(+)$ & $\begin{array}{l}\mathrm{MS}^{2}[479]: 304(15.6), 303(100) \\
\operatorname{MS}^{3}[479 \rightarrow 303]: 285 \text { (33.1), } 257(71.4), 229(100)\end{array}$ & Delphinidin glucuronide & Cooke et al. (2006) \\
\hline 35 & 7.2 & 865 & $\begin{array}{l}\mathrm{MS}^{2}[865]: 739(33), 713(24.1), 695(100), 577(39.9), 543(56), \\
407(40), 289(16.3) \\
\mathrm{MS}^{3}[865 \rightarrow 695]: 543(100), 525(54.3), 451(25.8), 408(27.3), \\
407(86.9) \\
\mathrm{MS}^{4}[865 \rightarrow 695 \rightarrow 543]: 525(100), 499(14.9), 392(30.9), 391 \\
(18), 255(14.5)\end{array}$ & Procyanidin trimer & $\begin{array}{l}\text { Ruiz et al. (2005); } \\
\text { Tomás-Barberán } \\
\text { et al. (2001) }\end{array}$ \\
\hline 39 & 7.7 & 577 & $\begin{array}{l}\mathrm{MS}^{2}[577]: 451(19.8), 426(19), 425 \text { (100), } 407 \text { (74.7), } 289 \\
(12.4), 287(13.1) \\
\text { MS }^{3}[577 \rightarrow 425]: 408(22.3), 407(100) \\
\text { MS }^{4}[577 \rightarrow 425 \rightarrow 407]: 285(100), 283(28.5), 281(63.8), 257 \\
(35.4), 256(28.4)\end{array}$ & Procyanidin dimer & $\begin{array}{l}\text { Ruiz et al. (2005); } \\
\text { Tomás-Barberán } \\
\text { et al. (2001) }\end{array}$ \\
\hline 41 & 8.1 & 607 & $\begin{array}{l}\mathrm{MS}^{2}[607]: 321(8.1), 285(100) \\
\mathrm{MS}^{3}[607 \rightarrow 285]: 257(28.3), 241(26.3), 229(25), 213(44.1) \\
151(100) \\
\operatorname{MS}^{4}[607 \rightarrow 285 \rightarrow 151]: 107(100)\end{array}$ & Kaempferol derivative & - \\
\hline 43 & 8.4 & 593 & $\begin{array}{l}\mathrm{MS}^{2}[593]: 307(12), 286(13), 285(100) \\
\operatorname{MS}^{3}[593 \rightarrow 285]: 267(28.9), 257(82.3), 229(46.1), 223(50.7) \\
151(100) \\
\operatorname{MS}^{4}[593 \rightarrow 285 \rightarrow 151]: 169(52.4), 107(100)\end{array}$ & Kaempferol 3-rutinoside & Del Rio et al. (2004) \\
\hline
\end{tabular}


Table 4 (Continued)

\begin{tabular}{|c|c|c|c|c|c|}
\hline No & $t_{R}(\min )$ & {$[\mathrm{M}-\mathrm{H}]^{-}(m / z)$} & HPLC-DAD-MS $; m / z$ (\% base peak) & Assigned identity & Ref. \\
\hline 46 & 9.4 & 563 & $\begin{array}{l}\mathrm{MS}^{2}[563]: 281(100), 279(9.7), 237(19.6), 193(12), 161(9.3) \\
\mathrm{MS}^{3}[563 \rightarrow 281]: 237(48.1), 193(57.6), 191(17.8), 161(44.6) \\
109(100)\end{array}$ & Unknown & - \\
\hline 49 & 9.7 & 477 & $\begin{array}{l}\mathrm{MS}^{2}[477]: 302(16.4), 301(100) \\
\mathrm{MS}^{3}[477 \rightarrow 301]: 273(12), 257(7), 179(100), 151(85.7) \\
\mathrm{MS}^{4}[477 \rightarrow 301 \rightarrow 151]: 170(13.4), 169(46.7), 107(100), 83 \\
(6.2), 65(11.1) \\
\mathrm{MS}^{4}[477 \rightarrow 301 \rightarrow 179]: 169(72.2), 152(35.1), 151(100)\end{array}$ & Quercetin-3-O-glucuronide & $\begin{array}{l}\text { Downey and } \\
\text { Rochfort (2008) }\end{array}$ \\
\hline 50 & 9.9 & 463 & $\begin{array}{l}\operatorname{MS}^{2}[463]: 288(12.4), 287(100), 151(18) \\
\operatorname{MS}^{3}[463 \rightarrow 287]: 151(100), 135(4.7) \\
\operatorname{MS}^{4}[463 \rightarrow 287 \rightarrow 151]: 107(100)\end{array}$ & Eriodictyol glucuronide & Fabre et al. (2001) \\
\hline 53 & 10.9 & $373(+)$ & $\begin{array}{l}\mathrm{MS}^{2}[373]: 211(100), 193(65.0), 175(23.7), 135(41.1), 119 \\
(26.8) \\
\mathrm{MS}^{3}[373 \rightarrow 211]: 193(100), 175(65.3), 135(41.7), 119(72.1) \\
109(20.0) \\
\text { MS }^{4}[373 \rightarrow 211 \rightarrow 193]: 175(100), 135(36.4), 121(16.5), 119 \\
(73.6)\end{array}$ & Hydroferuloylglucose & Ma et al. (2007) \\
\hline 61 & 13.8 & 539 & $\begin{array}{l}\mathrm{MS}^{2}[539]: 377(21.2), 327(15.3), 307(61.8), 275(100) \\
\text { MS }^{3}[539 \rightarrow 275]: 139(100), 95(83.8)\end{array}$ & Oleuropein & $\begin{array}{l}\text { Bianco et al. } \\
\text { (2001); Fu et al. } \\
(2010)\end{array}$ \\
\hline 62 & 13.8 & 359 & $\begin{array}{l}\operatorname{MS}^{2}[359]: 197(20.4), 179(21.8), 161(100) \\
\operatorname{MS}^{2}[359 \rightarrow 161]: 134(54.3), 133(100)\end{array}$ & Rosmarinic acid & Liu et al. (2007) \\
\hline 63 & 14 & 447 & $\begin{array}{l}\mathrm{MS}^{2}[447]: 272(34.5), 271(100), 175(45.8) \\
\operatorname{MS}^{3}[447 \rightarrow 271]: 177(41.1), 166(16.5), 151(100), 125(10.3) \\
93(13.3) \\
\operatorname{MS}^{4}[447 \rightarrow 271 \rightarrow 151]: 107(100)\end{array}$ & Naringenin glucuronide & $\begin{array}{l}\text { Sánchez-Rabaneda } \\
\text { et al. (2004) }\end{array}$ \\
\hline 75 & 19.6 & 493 & $\begin{array}{l}\mathrm{MS}^{2}[493]: 448(27.2), 447(100) \\
\operatorname{MS}^{3}[493 \rightarrow 447]: 315(100), 191(15), 161(86.7), 149(23.6), \\
131(15.4) \\
\text { MS }^{4}[493 \rightarrow 447 \rightarrow 315]: 161(100), 143(12.3), 113(10.8), 101 \\
(10.3),\end{array}$ & Unknown & - \\
\hline 78 & 22.8 & 543 & $\begin{array}{l}\mathrm{MS}^{2}[543]: 255(100) \\
\mathrm{MS}^{3}[543 \rightarrow 255]: 211(11.3), 193 \text { (52.3), } 175(100), 97(27.5), \\
81(12.2) \\
\mathrm{MS}^{4}[543 \rightarrow 255 \rightarrow 175]: 157(94), 113(59.8), 103(81.1), 83 \\
(24.2), 71(100)\end{array}$ & Catechin derivative & - \\
\hline 88 & 30.9 & 625 & $\begin{array}{l}\operatorname{MS}^{2}[625]: 602(100), 464(12.5), 463(60.8) \\
\operatorname{MS}^{3}[625 \rightarrow 602]: 463(100) \\
\operatorname{MS}^{4}[625 \rightarrow 602 \rightarrow 463]: 397(100)\end{array}$ & Unknown & - \\
\hline 91 & 31.5 & 327 & $\begin{array}{l}\operatorname{MS}^{2}[327]: 292(15.5), 291(33.6), 229(83.4), 211(33.1), 171 \\
(100) \\
\text { MS }^{3}[327 \rightarrow 229]: 211(100), 209(82.4), 125(23.1) \\
\text { MS }^{4}[327 \rightarrow 229 \rightarrow 209]: 165(100), 95(15.9)\end{array}$ & $\begin{array}{l}\text { Oxo-dihydroxy-octadecenoic } \\
\text { acid }\end{array}$ & $\begin{array}{l}\text { Levandi et al. } \\
\text { (2009); Van } \\
\text { Hoyweghen et al. } \\
\text { (2013) }\end{array}$ \\
\hline 96 & 34.3 & 329 & $\begin{array}{l}\operatorname{MS}^{2}[329]: 311(24.8), 293(22.4), 229(100), 211(73.6), 193 \\
(7.1), 171(22.8) \\
\operatorname{MS}^{3}[329 \rightarrow 229]: 211(85.6), 209(36.7), 167(27.2), 127 \text { (49.5), } \\
125(100)\end{array}$ & Trihydroxy-octadecenoic acid & $\begin{array}{l}\text { Van Hoyweghen } \\
\text { et al. (2014) }\end{array}$ \\
\hline 98 & 36.4 & 503 & $\begin{array}{l}\mathrm{MS}^{2}[503]: 486(29), 485(100), 453(41.5) \\
\mathrm{MS}^{3}[503 \rightarrow 485]: 453(100), 439(83.9), 403(90) \\
\mathrm{MS}^{4}[503 \rightarrow 485 \rightarrow 453]: 423(100), 409(25.5)\end{array}$ & Unknown & - \\
\hline 101 & 38.9 & 503 & $\begin{array}{l}\mathrm{MS}^{2}[503]: 486(29.3), 485(100) \\
\mathrm{MS}^{3}[503 \rightarrow 485]: 441(100), 439(32.5), 421(89.5), 403(68.4) \\
\mathrm{MS}^{4}[503 \rightarrow 485 \rightarrow 441]: 421(100), 419(53.8) \\
\mathrm{MS}^{4}[503 \rightarrow 485 \rightarrow 421]: 403(100), 393(90.6)\end{array}$ & Unknown & - \\
\hline 103 & 40.2 & 503 & $\begin{array}{l}\mathrm{MS}^{2}[503]: 486(25), 485(100) \\
\mathrm{MS}^{3}[503 \rightarrow 485]: 453(32.6) 439(100), 421(79.2) \\
\mathrm{MS}^{4}[503 \rightarrow 485 \rightarrow 439]: 423(61.1), 421(24.1), 407(100)\end{array}$ & Unknown & \\
\hline 105 & 43.1 & 487 & $\begin{array}{l}\operatorname{MS}^{2}[487]: 469(100) \\
\operatorname{MS}^{3}[487 \rightarrow 469]: 437(66.8), 435(100), 424(17.1), 392(34.8) \\
\operatorname{MS}^{4}[487 \rightarrow 469 \rightarrow 435]: 375(100)\end{array}$ & Unknown & - \\
\hline 107 & 44.6 & 487 & $\begin{array}{l}\mathrm{MS}^{2}[487]: 470(30.3), 469(100), 467(12.3) \\
\mathrm{MS}^{3}[487 \rightarrow 469]: 423(100), 421(21.4), 406(34.4), 393(15.2) \\
389(26.4) \\
\mathrm{MS}^{4}[487 \rightarrow 469 \rightarrow 423]: 407(34.4), 405(69.4), 403(42), 393 \\
(24.9), 389(100)\end{array}$ & Unknown & - \\
\hline
\end{tabular}

\subsection{Potential applications of the main phenolic compounds identified}

The main goal of this research was to find potential applications to the discarded vegetal material during forest cleaning tasks, particularly to leaves with high phenolic contents, which may represent a source of valuable bioactive compounds.

In O. europaea, the most abundant compound was oleuropein, as well as different derivatives or isomers. Due to its beneficial biological activities, the possibility of preparing oleuropein-enriched 
functional foods has been recently reported (Zoidou et al., 2014). Hence, discarded leaves from O.europaea may represent a source of oleuropein with applications in the food industry.

I. perado presented high levels of mono- and di-caffeoylquinic acids. Dried leaves of $I$. paraguariensis are used to prepare infusions rich in phenolic acids (Bastos et al., 2007), so further experiments could confirm the usefulness of $I$. perado for similar purposes.

Finally, different flavonoid glycosides, and procyanidin dimers and trimers were found in $H$. excelsa and $C$. arborea. However, the levels of these compounds were not as high as those found in $O$. europaea or I. perado, so further research would be necessary to confirm the potential uses of leaves from these plants.

\section{Conclusion}

In this work, a report on the phenolic composition of leaves of several relevant plants endemic to Madeira Archipelago is presented for the first time. Using HPLC-ESI-MS ${ }^{\mathrm{n}}$, different families of compounds were identified.

O. europaea ssp. cerasiformis extract was mainly constituted by oleuropein derivatives, like other varieties of 0 . europaea, and a simple flavonoid pattern of luteolin, apigenin and quercitin glucosides. A kaempferol triglycoside was the differentiating component, being previously reported in tea and citrus but not in Olea ssp. Caffeic acid derivatives and triterpenoid saponins were the main components of Ilex perado. Clethra arborea most relevant components in the methanolic extract were catechin derivatives, dimers and trimers, type B procyanidins, and flavonoid glucuronides. The identification of components of Heberdenia excelsa was not fully achieved and several components remain unknown, although several glycosylated flavonoids were identified. Phenylethanoids and isoflavoids were the differentiating components of this extract.

In total, over 100 compounds were detected in the analyzed extracts. Taking into account that there are no bibliographic data available in scientific literature for this species of plants, more research will be carried out in our research group, focusing in the identification of some of the unknowns, and the isolation and bioevaluation of relevant components.

\section{Acknowledgments}

This research was supported by Fundação para a Ciência e a Tecnologia (FCT) with funds from the Portuguese Government (Project PEst-OE/QUI/UI0674/2011). The mass spectrometer used in this work is part of the Portuguese National Mass Spectrometry Network (Contract RNEMREDE/1508/REM/2005) and was purchased in the framework of the National Programme for Scientific Reequipment, with funds from POCI 2010 (FEDER) and FCT. EJLM also acknowledges the financial support from Campus de Excelencia Internacional Agroalimentario (ceiA3) and University of Jaén, from Spain.

\section{References}

Altiok, E., Bayçin, D., Bayraktar, O., Ülkü, S., 2008. Isolation of polyphenols from the extracts of olive leaves (Olea europaea L.) by adsorption on silk fibroin. Sep. Purif. Technol. 62, 342-348.

Barreca, D., Bellocco, E., Caristi, C., Leuzzi, U., Gattuso, G., 2011. Distribution of C- and O-glycosyl flavonoids, (3-hydroxy-3-methylglutaryl)glycosyl flavanones and furocoumarins in Citrus aurantium L. juice. Food Chem. 124, 576-582.

Bastos, D.H.M., Saldanha, L.A., Catharino, R.R., Sawaya, A.C.H.F., Cunha, I.B.S., Carvalho, P.O., Eberlin, M.N., 2007. Phenolic antioxidants identified by ESI-MS from yerba maté (Ilex paraguariensis) and green tea (Camelia sinensis) extracts. Molecules 12, 423-432.

Benavente-García, O., Castillo, J., Lorente, J., Ortuño, A., Del Rio, J.A., 2000. Antioxidant activity of phenolics extracted from Olea europaea L. leaves. Food Chem. 68, 457-462.
Bianco, A., Buiarelli, F., Cartoni, G., Coccioli, F., Muzzalupo, I., Polidori, A., Uccella, N., 2001. Analysis by HPLC-MS/MS of biophenolic components in olives and oils. Anal. Lett. 34, 1033-1051

Bravo, L., Goya, L., Lecumberri, E., 2007. LC/MS characterization of phenolic constituents of mate (Ilex paraguariensis, St. Hil.) and its antioxidant activity compared to commonly consumed beverages. Food Res. Int. 40, 393-405.

Briante, R., Patumi, M., Terenziani, S., Bismuto, E., Febbraio, F., Nucci, R., 2002. Olea europaea L. leaf extract and derivatives: antioxidant properties. J. Agric. Food Chem. 50, 4934-4940.

Brito, G., Loureiro, J., Lopes, T., Rodriguez, E., Santos, C., 2008. Genetic characterisation of olive trees from Madeira Archipelago using flow cytometry and microsatellite markers. Gen. Res. Crop Evol. 55, 657-664.

Brudzynski, K., Miotto, D., 2011. Honey melanoidins: analysis of the compositions of the high molecular weight melanoidins exhibiting radical-scavenging activity. Food Chem. 127, 1023-1030.

Cardoso, S.M., Guyot, S., Marnet, N., Lopes-da-Silva, J.A., Renard, C.M.G.C., Coimbra, M.A., 2005. Characterisation of phenolic extracts from olive pulp and olive pomace by electrospray mass spectrometry. J. Sci. Food Agric. 85, 21-32.

Carini, M., Maffei Facino, R., Aldini, G., Calloni, M., Colombo, L., 1998. Characterization of phenolic antioxidants from Mate (Ilex paraguayensis) by liquid chromatography/mass spectrometry and liquid chromatography/tandem mass spectrometry. Rapid Commun. Mass Spectrom. 12, 1813-1819.

Clifford, M.N., Johnston, K.L., Knight, S., Kuhnert, N., 2003. Hierarchical scheme for LC-MSn identification of chlorogenic acids. J. Agric. Food Chem. 51, 2900-2911.

Clifford, M.N., Knight, S., Kuhnert, N., 2005. Discriminating between the six isomers of dicaffeoylquinic acid by LC-MSn. J. Agric. Food Chem. 53, 3821-3832.

Clifford, M.N., Marks, S., Knight, S., Kuhnert, N., 2006a. Characterization by LC-MSn of four new classes of p-coumaric acid-containing diacyl chlorogenic acids in green coffee beans. J. Agric. Food Chem. 54, 4095-4101.

Clifford, M.N., Zheng, W., Kuhnert, N., 2006b. Profiling the chlorogenic acids of aster by HPLC-MSn. Phytochem. Anal. 17, 384-393.

Chen, X.-Q., Zan, K., Yang, J., Liu, X.-X., Mao, Q., Zhang, L., Lai, M.-X., Wang, Q., 2011. Quantitative analysis of triterpenoids in different parts of Ilex hainanensis, Ilex stewardii and Ilex pubescens using HPLC-ELSD and HPLC-MSn and antibacterial activity. Food Chem. 126, 1454-1459.

Cooke, D.N., Thomasset, S., Boocock, D.J., Schwarz, M., Winterhalter, P., Steward, W.P., Gescher, A.J., Marczylo, T.H., 2006. Development of analyses by highperformance liquid chromatography and liquid chromatography/tandem mass spectrometry of bilberry (Vaccinium myrtilus) anthocyanins in human plasma and urine. J. Agric. Food Chem. 54, 7009-7013.

Damak, N., Bouaziz, M., Ayadi, M., Sayadi, S., Damak, M., 2008. Effect of the maturation process on the phenolic fractions, fatty acids, and antioxidant activity of the Chétoui olive fruit cultivar. J. Agric. Food Chem. 56, 1560-1566.

Dartora, N., De Souza, L.M., Santana-Filho, A.P., Iacomini, M., Valduga, A.T., Gorin P.A.J., Sassaki, G.L., 2011. UPLC-PDA-MS evaluation of bioactive compounds from leaves of Ilex paraguariensis with different growth conditions, treatments and ageing. Food Chem. 129, 1453-1461.

de Mejía, E.G., Chandra, S., Ramírez-Mares, M., Wang, W., 2006. Catalytic inhibition of human DNA topoisomerase by phenolic compounds in Ardisia compressa extracts and their effect on human colon cancer cells. Food Chem. Toxicol. 44 1191-1203.

De Nino, A., Lombardo, N., Perri, E., Procopio, A., Raffaelli, A., Sindona, G., 1997. Direct identification of phenolic glucosides from olive leaf extracts by atmospheric pressure ionization tandem mass spectrometry. J. Mass Spectrom. 32, 533-541.

Del Rio, D., Stewart, A.J., Mullen, W., Burns, J., Lean, M.E.J., Brighenti, F., Crozier, A. 2004. HPLC-MSn analysis of phenolic compounds and purine alkaloids in green and black tea. J. Agric. Food Chem. 52, 2807-2815.

Downey, M.O., Rochfort, S., 2008. Simultaneous separation by reversed-phase high-performance liquid chromatography and mass spectral identification of anthocyanins and flavonols in Shiraz grape skin. J. Chromatogr. A 1201, 43-47.

Eyles, A., Jones, W., Riedl, K., Cipollini, D., Schwartz, S., Chan, K., Herms, D., Bonello, P., 2007. Comparative phloem chemistry of Manchurian (Fraxinus mandshurica) and two North American ash species (Fraxinus americana and Fraxinus pennsylvanica). J. Chem. Ecol. 33, 1430-1448.

Fabre, N., Rustan, I., de Hoffmann, E., Quetin-Leclercq, J., 2001. Determination of flavone, flavonol, and flavanone aglycones by negative ion liquid chromatography electrospray ion trap mass spectrometry. J. Am. Soc. Mass Spectrom. 12, 707-715

Fu, S., Arráez-Roman, D., Segura-Carretero, A., Menéndez, J.A., Menéndez-Gutiérrez, M.P., Micol, V., Fernández-Gutiérrez, A., 2010. Qualitative screening of phenolic compounds in olive leaf extracts by hyphenated liquid chromatography and preliminary evaluation of cytotoxic activity against human breast cancer cells Anal. Bioanal. Chem. 397, 643-654.

González-Paramás, A.M., Lopes Da Silva, F., Martín-López, P., Macz-Pop, G. González-Manzano, S., Alcalde-Eon, C., Pérez-Alonso, J.J., Escribano-Bailón, M.T. Rivas-Gonzalo, J.C., Santos-Buelga, C., 2006. Flavanol--anthocyanin condensed pigments in plant extracts. Food Chem. 94, 428-436.

Gouveia, S., Castilho, P.C., 2011. Antioxidant potential of Artemisia argentea L'Hé alcoholic extract and its relation with the phenolic composition. Food Res. Int. 44, 1620-1631.

Gouveia, S., Castilho, P.C., 2012. Helichrysum monizii Lowe: phenolic composition and antioxidant potential. Phytochem. Anal. 23, 72-83.

Gouveia, S.C., Castilho, P.C., 2009. Analysis of phenolic compounds from different morphological parts of Helichrysum devium by liquid chromatography with on-line UV and electrospray ionization mass spectrometric detection. Rapid Commun. Mass Spectrom. 23, 3939-3953. 
Gouveia, S.C., Castilho, P.C., 2010. Characterization of phenolic compounds in Helichrysum melaleucum by high-performance liquid chromatography with on-line ultraviolet and mass spectrometry detection. Rapid Commun. Mass Spectrom. 24, 1851-1868.

Guo, H., Liu, A.-H., Ye, M., Yang, M., Guo, D.-A., 2007. Characterization of phenolic compounds in the fruits of Forsythia suspensa by high-performance liquid chromatography coupled with electrospray ionization tandem mass spectrometry. Rapid Commun. Mass Spectrom. 21, 715-729.

Han, J., Ye, M., Qiao, X., Xu, M., Wang B.-r., Guo, D.-A., 2008. Characterization of phenolic compounds in the Chinese herbal drug Artemisia annua by liquid chromatography coupled to electrospray ionization mass spectrometry. J. Pharm. Biomed. Anal. 47, 516-525.

Ignat, I., Volf, I., Popa, V.I., 2011. A critical review of methods for characterisation of polyphenolic compounds in fruits and vegetables. Food Chem. 126, 1821-1835.

IUCN, 2013. IUCN Red List of Threatened Species. Version 2013.2, http://www.iucnredlist.org/ (accessed 29.04.14).

Jemai, H., Bouaziz, M., Sayadi, S., 2009. Phenolic composition, sugar contents and antioxidant activity of Tunisian sweet olive cuitivar with regard to fruit ripening. J. Agric. Food Chem. 57, 2961-2968.

Khan, M.N.I., Nizami, S.S., Khan, M.A., Ahmed, Z., 1993. New saponins from Mangifera indica. J. Nat. Prod. 56, 767-770.

Laguerre, M., López Giraldo, L.J., Piombo, G., Figueroa-Espinoza, M.C., Pina, M. Benaissa, M., Combe, A., Rossignol Castera, A., Lecomte, J., Villeneuve, P., 2009. Characterization of olive-leaf phenolics by esi-ms and evaluation of their antioxidant capacities by the cat assay. J. Am. Oil Chem. Soc. 86, 1215-1225.

Levandi, T., Püssa, T., Vaher, M., Toomik, P., Kaljurand, M., 2009. Oxidation products of free polyunsaturated fatty acids in wheat varieties. Eur. J. Lipid Sci. Technol. $111,715-722$.

Liu, A.-H., Guo, H., Ye, M., Lin, Y.-H., Sun, J.-H., Xu, M., Guo, D.-A., 2007. Detection, characterization and identification of phenolic acids in Danshen using high-performance liquid chromatography with diode array detection and electrospray ionization mass spectrometry. J. Chromatogr. A 1161, 170-182.

Ma, C., Xiao, S.-y., Li, Z.-g., Wang, W., Du, L.-j., 2007. Characterization of active phenolic components in the ethanolic extract of Ananas comosus L. leaves using high-performance liquid chromatography with diode array detection and tandem mass spectrometry. J. Chromatogr. A 1165, 39-44.

Mateos-Martín, M.L., Fuguet, E., Quero, C., Pérez-Jiménez, J., Torres, J.L., 2012. New identification of proanthocyanidins in cinnamon (Cinnamomum zeylanicum L.) using MALDI-TOF/TOF mass spectrometry. Anal. Bioanal. Chem. 402, 1327-1336.

Mylonaki, S., Kiassos, E., Makris, D.P., Kefalas, P., 2008. Optimisation of the extraction of olive (Olea europaea) leaf phenolics using water/ethanol-based solvent systems and response surface methodology. Anal. Bioanal. Chem. 392 977-985.

Obied, H.K., Bedgood Jr., D.R., Prenzler, P.D., Robards, K., 2007. Chemical screening of olive biophenol extracts by hyphenated liquid chromatography. Anal. Chim. Acta 603, 176-189.

Pereira, A.P., Ferreira, I.C.F.R., Marcelino, F., Valentão, P., Andrade, P.B., Seabra, R Estevinho, L., Bento, A., Pereira, J.A., 2007. Phenolic compounds and antimicrobial activity of olive (Olea europaea L. Cv. Cobrançosa) leaves. Molecules 12 1153-1162.

Peres, R.G., Tonin, F.G., Tavares, M.F.M., Rodriguez-Amaya, D.B., 2013. HPLC DAD-ESI/MS identification and quantification of phenolic compounds in ilex paraguariensis beverages and on-line evaluation of individual antioxidant activity. Molecules 18, 3859-3871.

Prasain, J.K., Jones, K., Kirk, M., Wilson, L., Smith-Johnson, M., Weaver, C., Barnes, S., 2003. Profiling and quantification of isoflavonoids in kudzu dietary supplements by high-performance liquid chromatography and electrospray ionization tandem mass spectrometry. J. Agric. Food Chem. 51, 4213-4218.

Quirantes-Piné, R., Lozano-Sánchez, J., Herrero, M., Ibáñez, E., Segura-Carretero, A. Fernández-Gutiérrez, A., 2013. HPLC-ESI-QTOF-MS as a powerful analytical tool for characterising phenolic compounds in olive-leaf extracts. Phytochem. Anal. 24, 213-223.

Rivera, D.O.C., 1995. The ethnopharmacology of Madeira and Porto Santo Islands, a review. J. Ethnopharmacol. 46, 21.

Rockenbach, I.I., Jungfer, E., Ritter, C., Santiago-Schübel, B., Thiele, B., Fett, R., Galensa, R., 2012. Characterization of flavan-3-ols in seeds of grape pomace by CE, HPLCDAD-MS $n$ and LC-ESI-FTICR-MS. Food Res. Int. 48, 848-855.

Ruiz, D., Egea, J., Gil, M.I., Tomás-Barberán, F.A., 2005. Characterization and quantitation of phenolic compounds in new apricot (Prunus armeniaca L.) varieties. J. Agric. Food Chem. 53, 9544-9552.

Sánchez-Rabaneda, F., Jáuregui, O., Lamuela-Raventós, R.M., Viladomat, F., Bastida J., Codina, C., 2004. Qualitative analysis of phenolic compounds in apple pomace using liquid chromatography coupled to mass spectrometry in tandem mode. Rapid Commun. Mass Spectrom. 18, 553-563.

Savarese, M., De Marco, E., Sacchi, R., 2007. Characterization of phenolic extracts from olives (Olea europaea cv. Pisciottana) by electrospray ionization mass spectrometry. Food Chem. 105, 761-770.

Schütz, K., Kammerer, D.R., Carle, R., Schieber, A., 2005. Characterization of phenolic acids and flavonoids in dandelion (Taraxacum officinale WEB. ex WIGG.) root and herb by high-performance liquid chromatography/electrospray ionization mass spectrometry. Rapid Commun. Mass Spectrom. 19, 179-186.

Sosa, P.A., González-González, E.A., González-Pérez, M.A., Pérez de Paz, P.L., 2013. Contrasting patterns of genetic differentiation in macaronesian lineages of ilex (aquifoliaceae). Bot. J. Linn. Soc. 173, 258-268.

Suárez, M., Valls, R.M., Romero, M.P., Maclà, A., Fernández, S., Giralt, M., Solà, R. Motilva, M.J., 2011. Bioavailability of phenols from a phenol-enriched olive oil Br. J. Nutr. 106, 1691-1701.

Tomás-Barberán, F.A., Gil, M.I., Cremin, P., Waterhouse, A.L., Hess-Pierce, B., Kader A.A., 2001. HPLC-DAD-ESIMS analysis of phenolic compounds in nectarines, peaches, and plums. J. Agric. Food Chem. 49, 4748-4760.

Truchado, P., Vit, P., Ferreres, F., Tomas-Barberan, F., 2011. Liquid chromatography-tandem mass spectrometry analysis allows the simultaneous characterization of C-glycosyl and O-glycosyl flavonoids in stingless bee honeys. J. Chromatogr. A 1218, 7601-7607.

Urpi-Sarda, M., Garrido, I., Monagas, M.a., Goímez-Cordovés, C., Medina-Remoín, A. Andres-Lacueva, C., Bartolomé, B.a., 2009a. Profile of plasma and urine metabolites after the intake of almond [Prunus dulcis (Mill.) D.A. Webb] polyphenols in humans. J. Agric. Food Chem. 57, 10134-10142.

Urpi-Sarda, M., Monagas, M., Khan, N., Llorach, R., Lamuela-Raventós, R.M., Jáuregui, O., Estruch, R., Izquierdo-Pulido, M., Andrés-Lacueva, C., 2009b. Targeted metabolic profiling of phenolics in urine and plasma after regular consumption of cocoa by liquid chromatography-tandem mass spectrometry. J. Chromatogr. A $1216,7258-7267$

Van Hoyweghen, L., De Bosscher, K., Haegeman, G., Deforce, D., Heyerick, A., 2014 In vitro inhibition of the transcription factor NF-кB and cyclooxygenase by bamboo extracts. Phytother. Res. 28, 224-230.

Ye, M., Yan, Y., Guo, D.A., 2005. Characterization of phenolic compounds in the Chinese herbal drug $\mathrm{Tu}-\mathrm{Si}-\mathrm{Zi}$ by liquid chromatography coupled to electrospray ionization mass spectrometry. Rapid Commun. Mass Spectrom. 19, 1469-1484.

Zoidou, E., Magiatis, P., Melliou, E., Constantinou, M., Haroutounian, S., Skaltsounis A.L., 2014. Oleuropein as a bioactive constituent added in milk and yogurt. Food Chem. 158, 319-324. 